\title{
Interactive and Noninteractive Zero Knowledge are Equivalent in the Help Model ${ }^{\star}$
}

\author{
André Chailloux ${ }^{1, \star \star}$, Dragos Florin Ciocan $^{2, * * *}$, Iordanis Kerenidis $^{1, * *}$, \\ and Salil Vadhan ${ }^{2, \star \star \star}$ \\ ${ }^{1}$ LRI, Université Paris-Sud, Orsay, France \\ \{andre.chailloux, jkeren\}@lri.fr \\ 2 School of Engineering and Applied Sciences, Harvard University, Cambridge, MA \\ $\{$ ciocan, salil\}@eecs. harvard.edu
}

\begin{abstract}
We show that interactive and noninteractive zero-knowledge are equivalent in the 'help model' of Ben-Or and Gutfreund (J. Cryptology, 2003). In this model, the shared reference string is generated by a probabilistic polynomial-time dealer who is given access to the statement to be proven. Our results do not rely on any unproven complexity assumptions and hold for statistical zero knowledge, for computational zero knowledge restricted to AM, and for quantum zero knowledge when the help is a pure quantum state.
\end{abstract}

Keywords: cryptography, computational complexity, noninteractive zero-knowledge proofs, commitment schemes, Arthur-Merlin games, quantum zero knowledge.

\section{Introduction}

Zero-knowledge proofs 4 are protocols whereby a prover can convince a verifier that some assertion is true with the property that the verifier learns nothing else from the protocol. This remarkable property is easily seen to be impossible for the classical notion of a proof system, where the proof is a single string sent from the prover to the verifier, as the proof itself constitutes 'knowledge' that the verifier could not have feasibly generated on its own (assuming NP $\nsubseteq$ BPP). Thus zero-knowledge proofs require some augmentation to the classical model for proof systems.

The original proposal of Goldwasser, Micali, and Rackoff [4 augments the classical model with both randomization and multiple rounds of interaction between

* Preliminary versions of this work previously appeared on the Cryptology ePrint Archive [12], and in the second author's undergraduate thesis 3 .

** Supported in part by ACI Securité Informatique SI/03 511 and ANR AlgoQP grants of the French Ministry and in part by the European Commission under the Integrated Project Qubit Applications (QAP) funded by the IST directorate as Contract Number 015848.

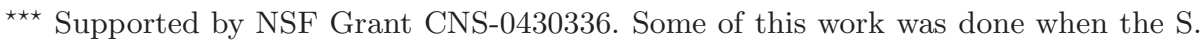
Vadhan was visiting U.C. Berkeley, supported by a Guggenheim Fellowship and the Miller Institute for Basic Research in Science. 
the prover and the verifier, leading to what are called interactive zero-knowledge proofs, or simply zero-knowledge proofs. An alternative model, proposed by Blum, Feldman, and Micali [5]6, augments the classical model with a set-up in which a trusted dealer randomly generates a reference string that is shared between the prover and verifier. After this reference string is generated, the proof consists of a single message from the prover to the verifier. Thus, these are referred to as noninteractive zero-knowledge proofs. Since their introduction, there have been many constructions of both interactive and noninteractive zero-knowledge proofs, and both models have found numerous applications in the construction of cryptographic protocols.

It is natural to ask what is the relation between these two models, that is:

Can every assertion that can be proven with an interactive zero-knowledge proof also be proven with a noninteractive zero-knowledge proof?

Our main result is a positive answer to this question in the 'help model' of Ben-Or and Gutfreund 7], where the dealer is given access to the statement to be proven when generating the reference string. We hope that this will serve as a step towards answering the above question for more standard models of noninteractive zero knowledge, such as the common reference string model and the public parameter model.

\subsection{Models of Zero Knowledge}

Interactive Zero Knowledge. Recall that an interactive proof system 4 for a problem $\Pi$ is an interactive protocol between a computationally unbounded prover $P$ and a probabilistic polynomial-time verifier $V$ that satisfies the following two properties:

- Completeness: if $x$ is a YES instance of $\Pi$, then the $V$ will accept with high probability after interacting with the $P$ on common input $x$.

- Soundness: if $x$ is a No instance of $\Pi$, then for every (even computationally unbounded) prover strategy $P^{*}, V$ will reject with high probability after interacting with $P^{*}$ on common input $x$.

Here, we consider problems $\Pi$ that are not only languages, but also ones that are promise problems, meaning that some inputs can be neither YES nor NO instances, and we require nothing of the protocol on such instances. (Put differently, we are 'promised' that the input $x$ is either a YES or a NO instance.) We write IP for the class of promise problems possessing interactive proof systems.

As is common in complexity-theoretic studies of interactive proofs and zero knowledge, we allow the honest prover $P$ to be computationally unbounded, and require soundness to hold against computationally unbounded provers. However, cryptographic applications of zero-knowledge proofs typically require an honest prover $P$ that can be implemented in probabilistic polynomial-time given a witness of membership for $x$, and it often suffices for soundness to hold only 
for polynomial-time prover strategies $P^{*}$ (leading to interactive argument systems [8]). It was recently shown how to extend the complexity-theoretic studies of interactive zero knowledge proofs to both polynomial-time honest provers 9 , and to argument systems [10; we hope that the same will eventually happen for noninteractive zero knowledge.

Intuitively, we say that an interactive proof system is zero knowledge if the verifier 'learns nothing' from the interaction other than the fact that the assertion being proven is true, even if the verifier deviates from the specified protocol. Formally, we require that there is an efficient algorithm, called the simulator, that can simulate the verifier's view of the interaction given only the YES instance $x$ and no access to the prover $P$. The most general notion, computational zero knowledge or just zero knowledge, requires this to hold for all polynomialtime cheating verifier strategies (and the simulation should be computationally indistinguishable from the verifier's view). A stronger notion, statistical zero knowledge, requires security against even computationally unbounded verifier strategies (and the simulation should be statistically indistinguishable from the verifier's view). We write ZK (resp., SZK) to denote the class of promise problems possessing computational (resp., statistical) zero-knowledge proof systems.

Noninteractive Zero Knowledge. For noninteractive zero knowledge [5]6, we introduce a trusted third party, the dealer, who randomly generates a reference string that is provided to both the prover and verifier. After that, the prover sends a single message to the verifier, who decides whether to accept or reject. Completeness and soundness are defined analogously to interactive proofs, except that the probabilities are now also taken over the choice of the reference string. Computational and statistical zero knowledge are also defined analogously to the interactive case, except that now the reference string is also considered part of the verifier's view, and must also be simulated.

There are a number of variants of the noninteractive model, depending on the form of the trusted set-up performed by the dealer. In the original, common random string (crs) model proposed by Blum et al. [56], the reference string is simply a uniformly random string of polynomial length. This gives rise to the classes NIZK ${ }^{\mathrm{crs}}$ and NISZK ${ }^{\mathrm{crs}}$ of problems having noninteractive computational and statistical zero-knowledge proofs in the common random string model. A natural and widely used generalization is the public parameter model, where the reference string need not be uniform, but can be generated according to any polynomial-time samplable distribution. That is, we obtain the reference string by running a probabilistic polynomial-time dealer algorithm $D$ on input $1^{n}$, where $n$ is the length of statements to be proven (or the security parameter). This model gives rise to the classes NIZK ${ }^{\text {pub }}$ and NISZK ${ }^{\text {pub }}$.

A further generalization is the help model introduced by Ben-Or and Gutfreund 7]. In this model, the distribution of the reference string is allowed to depend on the statement $x$ being proven. That is, the reference string is generated by running a probabilistic polynomial-time dealer algorithm $D$ on input $x$. We denote the class of problems having computational (resp. statistical) zeroknowledge proofs in this model as NIZK ${ }^{\mathrm{h}}$ (resp., NISZK ${ }^{\mathrm{h}}$ ). This model does not 
seem to suffice for most cryptographic applications, but its study may serve as a stepping stone towards a better understanding of the more standard models of noninteractive zero knowledge mentioned above. Indeed, any characterizations of noninteractive zero knowledge in the help model already serve as upper bounds on the power of noninteractive zero knowledge in the common random string and public parameter models.

We remark that one can also consider protocols in which we allow both a trusted dealer and many rounds of interaction. The most general model allows both help and interaction, yielding the classes $\mathrm{ZK}^{\mathrm{h}}$ and $\mathrm{SZK}^{\mathrm{h}}$.

Quantum Interactive and Noninteractive Zero Knowledge. The definitions of interactive proofs and zero knowledge extend naturally to the quantum setting. A quantum interactive proof system ([1]) for a promise problem $\Pi$ is an interactive protocol between a computationally unbounded prover $P$ and a quantum polynomial-time verifier $V$ that satisfies completeness and soundness properties as in the classical case and where the interaction is via quantum messages.

For quantum zero knowledge [12, we require that the verifier's view (which consists of qubits) can be simulated by a quantum polynomial-time machine. QSZK denotes the class of promise problems possessing quantum statistical zeroknowledge proof systems. Kobayashi 13 defined quantum noninteractive zero knowledge by having a dealer generate and share a maximally entangled quantum state between the prover and verifier. We write QNISZK to denote the class of promise problems possessing such quantum noninteractive statistical zeroknowledge proof systems.

In this paper, we define two more variants of the quantum noninteractive model, depending on the form of the trusted help created by the dealer. When the help is a pure quantum state that depends on the statement $x$ being proven we have the class QNISZK ${ }^{\mathrm{h}}$. When the help is a mixed quantum state that depends on $x$, we have the class QNISZK ${ }^{\mathrm{mh}}$. Last, the class QSZK ${ }^{\mathrm{h}}$ refers to protocols where we allow both a pure quantum help and interaction.

\subsection{Previous Work}

Recall that we are interested in the relationship between the interactive zeroknowledge classes ZK and SZK and their various noninteractive counterparts, which we will denote by NIZK and NISZK when we do not wish to specify the model. That is, for a given model of noninteractive zero knowledge, we ask: Does $\mathrm{ZK}=\mathrm{NIZK}$ and $\mathrm{SZK}=\mathrm{NISZK} ?$

$\boldsymbol{Z K}$ vs. $\boldsymbol{N I Z K}$. A first obstacle to proving equality of ZK and NIZK is that NIZK is a subset of AM, the class of problems having constant-round interactive proof systems [14 15, whereas ZK may contain problems outside of AM. So, instead of asking whether ZK = NIZK, we should instead ask if ZK $\cap \mathrm{AM}=\mathrm{NIZK}$.

Indeed, this equality is known to hold under complexity assumptions. If oneway permutations exist, then it is known that ZK $=$ IP 161718 and NIZK $^{\mathrm{crs}}=$ $\mathrm{AM}$ [19], and thus $\mathrm{ZK} \cap \mathrm{AM}=\mathrm{NIZK}^{\mathrm{crs}}=\mathrm{NIZK}^{\mathrm{pub}}=\mathrm{NIZK}^{\mathrm{h}}$. (In fact, if we 
replace NIZK $^{\text {crs }}$ with NIZK ${ }^{\text {pub }}$, these results hold assuming the existence of any one-way function $20 \mid 212223$.$) .) Thus, for computational zero knowledge, the$ interesting question is whether we can prove that $\mathrm{ZK} \cap \mathrm{AM}=\mathrm{NIZK}$ unconditionally, without assuming the existence of one-way functions. To our knowledge, there have been no previous results along these lines.

$S Z K$ vs. NISZK. For relating SZK and NISZK, the class AM no longer is a barrier, because it is known that $\mathrm{SZK} \subseteq \mathrm{AM}[24$.

The relationship between SZK and NISZK was first addressed in the work of Goldreich et al. 25. There it was shown that SZK and NISZK' ${ }^{\text {crs }}$ have the 'same complexity' in the sense that SZK $=$ BPP iff NISZK ${ }^{\mathrm{crs}}=\mathrm{BPP}$. Moreover, it was proven that $\mathrm{SZK}=\mathrm{NISZK}^{\mathrm{crs}}$ iff $\mathrm{NISZK}^{\mathrm{crs}}$ is closed under complement.

In addition to introducing the help model, Ben-Or and Gutfreund 7 studied the relationship between NISZK $^{\mathrm{h}}$ and SZK. They proved that NISZK ${ }^{\mathrm{h}} \subseteq$ SZK (in fact that $\mathrm{SZK}^{\mathrm{h}}=\mathrm{SZK}$ ), and posed as an open question whether $\mathrm{SZK} \subseteq$ NISZK $K^{\mathrm{h}}$

\subsection{Our Results}

We show that interactive zero knowledge does in fact collapse to noninteractive zero knowledge in the help model, both for the computational case (restricted to $\mathrm{AM}$ ) and the statistical case:

Theorem 1. $\mathrm{ZK} \cap \mathrm{AM}=\mathrm{NIZK}^{\mathrm{h}}$.

Theorem 2. SZK $=$ NISZK $^{\mathrm{h}}$.

These results and their proofs yield new characterizations of the classes ZK and SZK. For example, we obtain a new complete problem for SZK, namely the NISZK $^{\mathrm{h}}$-complete problem given in 7]. Similarly, we obtain a new characterization of ZK, which amounts to a computational analogue of the NISZK ${ }^{\mathrm{h}}$-complete problem. As suggested in [7, these results can also be viewed as first steps towards collapsing interactive zero knowledge to noninteractive zero knowledge in the public parameter or common reference string model. For example, to show SZK = NISZK ${ }^{\text {crs }}$ (the question posed in [26]), it now suffices to show that $\mathrm{NISZK}^{\mathrm{h}}=$ NISZK $^{\mathrm{crs}}$.

As mentioned above, one can consider even more general classes $\mathrm{ZK}^{\mathrm{h}}$ and $\mathrm{SZK}^{\mathrm{h}}$ that incorporate both help and interaction. Ben-Or and Gutfreund [7] showed that $\mathrm{SZK}^{\mathrm{h}}=\mathrm{SZK}$. We prove an analogous result for computational zero knowledge:

Theorem 3. $\mathrm{ZK}^{\mathrm{h}}=\mathrm{ZK}$.

In the quantum setting, very little is known about the relation of interactive and noninteractive quantum zero knowledge. Here, we start by providing two complete problems for the class QNISZK. Then, we define two variants of quantum

\footnotetext{
${ }^{1}$ In fact, their conference paper 22 claimed to prove that $\mathrm{SZK}=\mathrm{NISZK}^{h}$, but this
} was retracted in the journal version 7 . 
noninteractive zero knowledge depending on the 'help' created by the dealer. In the case where the help is a pure quantum state that depends on the input $x$, we prove an analogue of Theorem 2

Theorem 4. $\mathrm{QNISZK}^{\mathrm{h}}=\mathrm{QSZK}=\mathrm{QSZK}^{\mathrm{h}}$.

In the case where the help is a mixed quantum state, we show that the class QNISZK $^{\mathrm{mh}}$ contains AM and hence is most probably larger than QSZK.

\subsection{Techniques}

Here we sketch the techniques underlying the forward inclusions in Theorems 1 and 2, showing that interactive zero knowledge is a subset of noninteractive zero knowledge in the help model.

We begin with the case of statistical zero knowledge. Our proof that SZK $\subseteq$ NISZK $^{\mathrm{h}}$ is similar to the approach suggested by Goldreich et al. [25] for showing that $\mathrm{SZK}=\mathrm{NISZK}^{\mathrm{crs}}$. They showed that this question boils down to proving that co-NISZK $^{\mathrm{crs}}=$ NISZK $^{\mathrm{crs}}$ or in other words that the complement of the NISZK $^{\text {crs }}$-complete problem ENTROPY APPROXIMATION belongs to NISZK ${ }^{\mathrm{crs}}$. Similarly, the core part of our proof is showing that co-NISZK ${ }^{\mathrm{crs}} \subseteq$ NISZK $^{\mathrm{h}}$, which then we use to deduce that $\mathrm{SZK} \subseteq \mathrm{NISZK}^{\mathrm{h}}$.

More specifically, our goal is to reduce the SZK-complete problem ENTROPY DifFERENCE (ED) to the NISZK-complete problem IMAGE INTERSECTION DENSITY (IID). Following [25, we start by reducing ED to several instances of ENTROPY Approximation (EA) and its complement $(\overline{\mathrm{EA}})$. We know that EA $\in$ NISZK $^{\mathrm{h}}$ since by definition NISZK ${ }^{\mathrm{crs}} \subseteq$ NISZK $^{\mathrm{h}}$. Next, inspired by Ben-Or and Gutfreund's attempt 22] to reduce ED to IID and relying on ideas from [2728], we prove that $\overline{\mathrm{EA}}$ also belongs to NISZK ${ }^{\mathrm{h}}$. Thus we obtain a reduction from ED to several instances of IID. We then conclude our proof by showing that NISZK ${ }^{\mathrm{h}}$ has enough boolean closure properties to combine these several instances into a single instance of IID. We establish these closure properties of NISZK ${ }^{\mathrm{h}}$ and IID using techniques developed in [27/29] to show boolean closure properties for interactive SZK.

In the case of computational zero knowledge, we prove that $\mathrm{ZK} \cap \mathrm{AM} \subseteq \mathrm{NIZK}^{\mathrm{h}}$ by using certain variants of commitment schemes. Recall that a commitment scheme is a two-stage interactive protocol between a sender and a receiver. In the commit stage, the sender 'commits' to a secret message $m$. In the reveal stage, the sender 'reveals' $m$ and tries to convince the verifier that it was the message committed to in the first stage. Commitments should be hiding, meaning that an adversarial receiver will learn nothing about $m$ in the commit stage, and binding, meaning that after the commit stage, an adversarial sender should not be able to successfully reveal two different messages (except with negligible probability). Each of these security properties can be either computational, holding against polynomial-time adversaries, or statistical, holding even for computationally unbounded adversaries. Commitments are a basic building block for zero-knowledge protocols, e.g. they are the main cryptographic primitive used in the constructions of zero-knowledge proofs for all of NP [16] and IP [1718]. 
A relaxed notion is that of instance-dependent commitment schemes 3013132. Here the sender and receiver are given an instance $x$ of some problem $\Pi$ as auxiliary input. We only require the scheme to be hiding if $x$ is a YES instance, and only require it to be binding if $x$ is a No instance. They are a relaxation of standard commitment schemes because we do not require hiding and binding to hold simultaneously. Still, as observed in 31, an instance-dependent commitment scheme for a problem $\Pi \in$ IP suffices to construct zero-knowledge proofs for $\Pi$ because the constructions of [1617 18] only use the hiding property for zero knowledge (which is only required on YES instances), and the binding property for soundness (which is only required on NO instances).

We show that a similar phenomenon holds for noninteractive zero knowledge in the help model: If a problem $\Pi \in \mathrm{AM}$ has a certain kind of instance-dependent commitment scheme, then $\Pi \in \mathrm{NIZK}^{\mathrm{h}}$. For this, the instance-dependent commitments naturally need to be noninteractive. On the other hand, they only need to be binding (on No instances) in case the sender is honest during the commit phase. (Our observation is that such commitments can be used to implement the hidden bits model of [19.)

Thus our task is reduced to showing that every problem in ZK has a noninteractive instance-dependent commitment scheme that is computationally hiding on YES instances and statistically binding for honest senders on NO instances. To prove this, we begin by observing that a problem $\Pi$ has such an instancedependent commitment scheme with statistical hiding if and only if $\Pi$ reduces to IID. Hence, the needed commitments already follow for all of SZK from our first result $\left(\mathrm{SZK} \subseteq \mathrm{NISZK}^{\mathrm{h}}\right)$. To obtain commitments for all of ZK, we use a characterization of ZK in terms of SZK and 'instance-dependent one-way functions' [33], and combine the instance-dependent commitment schemes we obtain from both SZK and the instance-dependent one-way functions.

An alternative construction of the instance-dependent commitments we need can be obtained by using the concurrent work of Ong and Vadhan 34. They showed that every problem in ZK (resp., SZK) has an instance-dependent commitment scheme that is computationally (resp., statistically) hiding on YES instances and statistically binding on NO instances. While their commitments are interactive, they can be made noninteractive if we assume that the sender is honest during the commit phase (by having the sender simulate both parties). Thus, our work can be viewed as a (substantial) simplification to their constructions for the case of honest senders.

\section{Definitions and Preliminaries}

\subsection{Promise Problems}

Promise problems are a more general variant of decision problems than languages. A promise problem $\Pi$ is a pair of disjoint sets of strings $\left(\Pi_{Y}, \Pi_{N}\right)$, where $\Pi_{Y}$ is the set of YES instances and $\Pi_{N}$ is the set of NO instances. The computational problem associated with any promise problem $\Pi$ is: given a string 
that is "promised" to lie in $\Pi_{Y} \cup \Pi_{N}$, decide whether it is in $\Pi_{Y}$ or $\Pi_{N}$. Reductions from one promise problem to another are natural extensions of reductions between languages. Namely, we say $\Pi$ reduces to $\Gamma$ (written $\Pi \preccurlyeq \Gamma$ ) if there exists a polynomial time computable function $f$ such that $x \in \Pi_{Y} \Rightarrow f(x) \in \Gamma_{Y}$ and $x \in \Pi_{N} \Rightarrow f(x) \in \Gamma_{N}$. We can also naturally extend the definitions of complexity classes by letting the properties of the strings in the languages be conditions on the YES instances, and properties of strings outside of the language be conditions on NO instances.

\subsection{Instance-Dependent Cryptographic Primitives}

Many of the objects that we will be constructing for use in our zero knowledge constructions will be instance dependent. Hence, we will modify common cryptographic primitives such as one-way functions by allowing them to be parametrized by some string $x$, such that the cryptographic properties will only be guaranteed to hold if $x$ is in some set $I$.

Definition 5. An instance-dependent function ensemble is a collection of functions $\mathcal{F}=\left\{f_{x}:\{0,1\}^{p(|x|)} \rightarrow\{0,1\}^{q(|x|}\right\}_{x \in\{0,1\}^{*}}$, where $p(\cdot)$ and $q(\cdot)$ are polynomials. $\mathcal{F}$ is polynomial-time computable if there exists a polynomial-time algorithm $F$ such that for all $x \in\{0,1\}^{*}$ and $y \in\{0,1\}^{p(|x|)}, F(x, y)=f_{x}(y)$.

Definition 6. An instance-dependent one-way function on $I$ is a polynomialtime instance-dependent function ensemble $\mathcal{F}=\left\{f_{x}:\{0,1\}^{p(|x|)} \rightarrow\right.$ $\left.\{0,1\}^{q(|x|}\right\}_{x \in\{0,1\}^{*}}$, such that for every nonuniform PPT $A$, there exists a negligible function $\varepsilon(\cdot)$ such that for all $x \in I$,

$$
\operatorname{Pr}\left[A\left(x, f_{x}\left(U_{p(|x|)}\right)\right) \in f_{x}^{-1}\left(f_{x}\left(U_{p(|x|)}\right)\right)\right] \leq \varepsilon(|x|)
$$

Definition 7. An instance-dependent probability ensemble on $I$ is a collection of random variables $\left\{X_{x}\right\}_{x \in\{0,1\}^{*}}$, where $X_{x}$ takes values in $\{0,1\}^{p(|x|)}$ for some polynomial $p$. We call such an ensemble samplable is there exists a probabilistic polynomial-time algorithm $M$ such that for every input $x, M(x)$ is distributed according to $X_{x}$.

Definition 8. Two instance-dependent probabilistic ensembles $\left\{X_{x}\right\}$ and $\left\{Y_{x}\right\}$ are computationally indistinguishable on $I \subset\{0,1\}^{*}$ if for every nonuniform PPT D, there exists a negligible $\varepsilon(\cdot)$ such that for all $x \in I$,

$$
\operatorname{Pr}\left[D\left(x, X_{x}\right)=1\right]-\operatorname{Pr}\left[D\left(x, Y_{x}\right)=1\right] \mid \leq \varepsilon(|x|)
$$

Similarly, we say $\left\{X_{x}\right\}$ and $\left\{Y_{x}\right\}$ are statistically indistinguishable on $I \subset$ $\{0,1\}^{*}$ if the above is required for all functions D. If $X_{x}$ and $Y_{x}$ are identically distributed for all $x \in I$, we say they are perfectly indistinguishable .

We will sometimes use the informal notation $X \stackrel{\mathrm{c}}{\equiv} Y$ to denote that ensembles $X$ and $Y$ are computationally indistinguishable. 
Definition 9. An instance-dependent pseudorandom generator on $I$ is a polynomial-time instance-dependent function ensemble $\mathcal{G}=\left\{G_{x}:\{0,1\}^{p(|x|)} \rightarrow\right.$ $\left.\{0,1\}^{q(|x|}\right\}$ such that $q(n)>p(n)$, and the probability ensembles $\left\{G_{x}\left(U_{p(|x|}\right)\right\}_{x}$ and $\left\{U_{q(|x|)}\right\}_{x}$ are computationally indistinguishable on $I$.

\subsection{Probability Distributions}

In this section, we define several tools that are useful for analysing properties of probability distributions.

Definition 10. The statistical difference between two random variables $X$ and $Y$ taking values in some domain $\mathcal{U}$ is defined as:

$$
\Delta(X, Y)=\max _{S \subset \mathcal{U}}|\operatorname{Pr}[X \in S]-\operatorname{Pr}[Y \in S]|=\frac{1}{2} \sum_{x \in \mathcal{U}}|\operatorname{Pr}[X=x]-\operatorname{Pr}[Y=x]|
$$

Definition 11. For an ordered pair of random variables $(X, Y)$, we define their disjointness to be:

$$
\operatorname{Disj}(X, Y)=\operatorname{Pr}_{X}[X \in \operatorname{Supp}(Y)]
$$

and we define their mutual disjointness:

$$
\operatorname{MutDisj}(X, Y)=\min (\operatorname{Disj}(X, Y), \operatorname{Disj}(Y, X)) .
$$

Note that disjointness is a more stringent measure of the disparity between two distributions than statistical difference. If two distributions have disjointness $\alpha$, then their statistical difference is at least $\alpha$. The converse, however, does not hold, since the two distributions could have statistical difference that is negligibly close to 1, yet have identical supports and mutual disjointness 0 .

Moreover, we can go from disjoint to mutually-disjoint distributions by the following lemma:

Lemma 12. [7.35] Given a pair of distributions $\left(X_{0}, X_{1}\right)$ with $n$ input gates, consider the following distributions:

$Y_{0}$ : Choose $r \stackrel{R}{\leftarrow}\{0,1\}^{n}, b \stackrel{R}{\leftarrow}\{0,1\}$, output $\left(X_{b}(r), b\right)$.

$Y_{1}$ : Choose $r \stackrel{R}{\leftarrow}\{0,1\}^{n}, b \stackrel{R}{\leftarrow}\{0,1\}$, output $\left(X_{b}(r), \bar{b}\right)$.

The following properties hold:

1. $\Delta\left(Y_{0}, Y_{1}\right)=\Delta\left(X_{0}, X_{1}\right)$

2. If $\left(X_{0}, X_{1}\right)$ is $\alpha$-disjoint, then $\left(Y_{0}, Y_{1}\right)$ is mutually $\frac{\alpha}{2}$-disjoint.

Tensoring Distributions. For random variables $X, Y$, we let $X \otimes Y$ be the random variable consisting of a sample of $X$ followed by an independent sample of $Y$. The $\otimes$ notation reflects the fact that the mass function of $X \otimes Y$ is the tensor product of the mass functions of $X$ and $Y$. When the independence is clear from context, we sometimes write $(X, Y)$ instead of $X \otimes Y . X^{\otimes k}$ is the random variable consisting of $k$ independent copies of $X$. 
Lemma $13([\mathbf{7}, 35])$. Given a parameter $k \in \mathbb{N}$ and the distributions $X_{1}, \ldots, X_{k}$ and $Y_{1}, \ldots, Y_{k}$, the pair $\left.(X, Y)=X_{1} \otimes \ldots \otimes X_{k}, Y_{1} \otimes \ldots \otimes Y_{k}\right)$ will satisfy the following properties:

1. $1-2 \exp \left(-k \delta^{2} / 2\right) \leq \Delta(X, Y) \leq k \delta$ where $\delta=\sum_{i \in[k]} \Delta\left(X_{i}, Y_{i}\right) / k$.

2. $\operatorname{MutDisj}(X, Y)=1-\prod_{i \in[k]}\left(1-\alpha_{i}\right)$, where $\alpha_{i}=\operatorname{MutDisj}\left(X_{i}, Y_{i}\right)$.

XORing Distributions. We define the XOR operator which acts on pairs of distributions and returns a pair of distributions. Given two pairs $\left(X_{0}, X_{1}\right)$ and $\left(X_{0}^{\prime}, X_{1}^{\prime}\right)$, with $n$ and $n^{\prime}$ input gates, respectively, $\operatorname{XOR}\left(\left(X_{0}, X_{1}\right),\left(X_{0}^{\prime}, X_{1}^{\prime}\right)\right)$ is defined by the circuits:

$Y_{0}$ : Choose $b \stackrel{R}{\leftarrow}\{0,1\}, r \stackrel{R}{\leftarrow}\{0,1\}^{n}, r^{\prime} \stackrel{R}{\leftarrow}\{0,1\}^{n^{\prime}}$, output $\left(X_{b}(r), X_{b}^{\prime}\left(r^{\prime}\right)\right)$.

$Y_{1}$ : Choose $b \stackrel{R}{\leftarrow}\{0,1\}, r \stackrel{R}{\leftarrow}\{0,1\}^{n}, r^{\prime} \stackrel{R}{\leftarrow}\{0,1\}^{n^{\prime}}$, output $\left(X_{b}(r), X_{b}^{\prime}\left(r^{\prime}\right)\right)$.

Lemma 14 (XOR Lemma [7, 35]). If $\left(Y_{0}, Y_{1}\right)=\operatorname{XOR}\left(\left(X_{0}, X_{1}\right),\left(X_{0}^{\prime}, X_{1}^{\prime}\right)\right)$, then the following properties hold:

1. $\Delta\left(Y_{0}, Y_{1}\right)=\Delta\left(X_{0}, X_{1}\right) \cdot \Delta\left(X_{0}^{\prime}, X_{1}^{\prime}\right)$.

2. $\operatorname{MutDisj}\left(Y_{0}, Y_{1}\right)=\operatorname{MutDisj}\left(X_{0}, X_{1}\right) \cdot \operatorname{MutDisj}\left(X_{0}^{\prime}, X_{1}^{\prime}\right)$.

By induction, the XOR Lemma implies the following method to decrease both statistical difference and mutual disjointness exponentially fast:

Lemma $15([7,35])$. Given circuits $X_{0}, X_{1}$ with $n$ input gates and a parameter $k$, consider the following pair:

$Y_{0}$ : Choose $\left(b_{1}, \ldots, b_{k}\right) \stackrel{R}{\leftarrow}\left\{\left(c_{1}, \ldots, c_{k}\right) \in\{0,1\}^{k}: c_{1} \oplus \ldots \oplus c_{k}=\right.$ $0\},\left(r_{1}, \ldots r_{k}\right) \stackrel{R}{\leftarrow}\{0,1\}^{k n}$, output $\left(X_{b_{1}}\left(r_{1}\right), \ldots, X_{b_{k}}\left(r_{k}\right)\right)$.

$Y_{1}$ : Choose $\left(b_{1}, \ldots, b_{k}\right) \stackrel{R}{\leftarrow}\left\{\left(c_{1}, \ldots, c_{k}\right) \in\{0,1\}^{k}: c_{1} \oplus \ldots \oplus c_{k}=\right.$ $1\},\left(r_{1}, \ldots r_{k}\right) \stackrel{R}{\leftarrow}\{0,1\}^{k n}$, output $\left(X_{b_{1}}\left(r_{1}\right), \ldots, X_{b_{k}}\left(r_{k}\right)\right)$.

The following properties hold:

1. $\Delta\left(Y_{0}, Y_{1}\right)=\Delta\left(X_{0}, X_{1}\right)^{k}$.

2. $\operatorname{MutDisj}\left(Y_{0}, Y_{1}\right)=\operatorname{MutDisj}\left(X_{0}, X_{1}\right)^{k}$.

Entropy and Hashing.

Definition 16. The entropy of a random variable $X$ is $\mathrm{H}(X)=$ $\mathrm{E}_{x \leftarrow X}\left[\log \frac{1}{\operatorname{Pr}[X=x]}\right]$. The conditional entropy of $X$ given $Y$ is

$\mathrm{H}(X \mid Y)=\underset{y \leftarrow Y}{\mathrm{E}}\left[\mathrm{H}\left(\left.X\right|_{Y=y}\right)\right]=\underset{(x, y) \leftarrow(X, Y)}{\mathrm{E}}\left[\log \frac{1}{\operatorname{Pr}[X=x \mid Y=y]}\right]=\mathrm{H}(X, Y)-\mathrm{H}(Y)$.

For entropy, it holds that for every $X, Y, \mathrm{H}(X \otimes Y)=\mathrm{H}(X)+\mathrm{H}(Y)$. More generally, if $(X, Y)^{\otimes^{k}}=\left(\left(X_{1}, Y_{1}\right), \ldots,\left(X_{k}, Y_{k}\right)\right)$, then $\mathrm{H}\left(\left(X_{1}, \ldots, X_{k}\right) \mid\left(Y_{1}, \ldots, Y_{k}\right)=\right.$ $k \cdot \mathrm{H}(X \mid Y)$. 
Definition 17. The relative entropy (Kullback-Liebler distance) between two distributions $X, Y$ is:

$$
\mathrm{KL}(X \mid Y)=\underset{x \leftarrow X}{\mathrm{E}}\left[\log \frac{\operatorname{Pr}[X=x]}{\operatorname{Pr}[Y=x]}\right]
$$

We denote by $\mathrm{H}_{2}(p)$ the binary entropy function, which is the entropy of a $\{0,1\}$-valued random variable with expectation $p . \mathrm{KL}_{2}(p, q)$ denotes the relative entropy between two $\{0,1\}$-value random variables with expectations $p$ and $q$.

Flat Distributions. Let $X$ a distribution with entropy $\mathrm{H}(X)$. Elements $x$ of $X$ such that $|\log \operatorname{Pr}[X=x]-\mathrm{H}(X)| \leq k$ are called $k$-typical. We say that $X$ is $\Delta$-flat if for every $t>0$ the probability that an element chosen from $X$ is $t \cdot \Delta$-typical is at least $1-2^{-t^{2}+1}$.

Lemma 18 (Flattening Lemma [36]). Let $X$ be a distribution encoded by a circuit with $n$ input gates. Then $X^{\otimes k}$ is $\sqrt{k} \cdot n$-flat.

Definition 19. A family $\mathcal{H}$ of functions from $A \rightarrow B$ is 2-universal if for every two elements $x \neq y \in A$ and $a, b \in B, \operatorname{Pr}_{h \in_{R} \mathcal{H}}[h(x)=a$ and $h(y)=b]=\frac{1}{|B|^{2}}$.

We write $\mathcal{H}_{n, m}$ to denote the 2-universal family from $\{0,1\}^{n}$ to $\{0,1\}^{m}$.

Lemma 20 (Leftover Hash Lemma [37]). Let $\mathcal{H}$ be a samplable family of 2universal hashing functions from $A \rightarrow B$. Suppose $X$ is a distribution on $A$ such that with probability at least $1-\delta$ over $x$ selected from $X, \operatorname{Pr}[X=x] \leq \epsilon /|B|$. Consider the following distribution:

$$
Z: \text { Choose } h \leftarrow \mathcal{H} \text { and } x \leftarrow X \text {, return }(h, h(x)) .
$$

Then, $\Delta(Z, \mathcal{U}) \leq O\left(\delta+\epsilon^{1 / 3}\right)$, where $\mathcal{U}$ is the uniform distribution on $\mathcal{H} \times B$.

\section{Interactive Zero Knowledge}

We consider a generalized version of interactive zero knowledge, introduced by Ben-Or and Gutfreund [7, in which the prover and the verifier have access to a help string output by a dealer algorithm that has access to the statement being proven. We will call this model of interactive zero knowledge the help model. Interactive zero-knowledge proofs are a special case of interactive zero-knowledge proofs in the help model.

We denote the three algorithms that make up an interactive zero-knowledge proof in the help model as $D, P$ and $V$. All three receive as input $x$, the statement being proven. The dealer selects the help string $\sigma \leftarrow D(x)$ and sends it to $P$ and $V$. $P$ and $V$ carry out an interactive protocol and, at the end of their interaction, they either output ACCEPT or REJECT. We call the transcript the sequence of messages which the triple $(D, P, V)$ computes. $(D, P, V)(x)$ denotes the random variable of the possible outcomes of the protocol, while $\langle D, P, V\rangle(x)$ denotes the verifier's view of the transcripts (where the probability space is over the random coins of $D, P$ and $V$ ). 
Definition $21\left(\mathrm{ZK}^{\mathrm{h}}, \mathrm{SZK}^{\mathrm{h}}[7]\right)$. A zero-knowledge proof system in the help model for a promise problem $\Pi$ is a triple of probabilistic algorithms $(D, P, V)$ (where $D$ and $V$ are polynomial time bounded), satisfying the following conditions:

1. Completeness. For all $x \in \Pi_{Y}, \operatorname{Pr}[(D, P, V)(x)=1] \geq \frac{2}{3}$, where the probability is taken over the coin tosses of $D, P$ and $V$.

2. Soundness. For all $x \in \Pi_{N}$ and every prover strategy $P^{*}$, $\operatorname{Pr}\left[\left(D, P^{*}, V\right)=1\right] \leq \frac{1}{3}$, where the probability is taken over the coin tosses of $D, P^{*}, V$.

3. Zero Knowledge. There exists a PPT $S$ such that the ensembles $\{\langle D, P, V\rangle)(x)\}_{x}$ and $\{S(x)\}_{x}$ are computationally indistinguishable on $\Pi_{Y}$.

If the ensembles are statistically indistinguishable, we call it a statistical zero knowledge proof system in the help model. ZK ${ }^{\mathrm{h}}$ (resp., SZK ${ }^{\mathrm{h}}$ ) is the class of promise problems possessing zero-knowledge (resp., statistical zero-knowledge) proof systems in the help model.

If the help string $\sigma$ is generated according to $D\left(1^{|x|}\right)$, we call the proof system an interactive zero-knowledge proof system in the public parameter model. The corresponding complexity class is $\mathrm{ZK}^{\mathrm{pub}}$ (resp., $\mathrm{SZK}^{\mathrm{pub}}$ ). If the help string $\sigma$ is generated from the uniform distribution on $\{0,1\}^{|x|}$, we call the proof system an interactive zero-knowledge proof system in the common random string model. The corresponding complexity class is $\mathrm{ZK}^{\mathrm{crs}}$ (resp., SZK ${ }^{\mathrm{crs}}$ ).

If we remove the dealer's help, the resulting proof system is said to be an interactive zero-knowledge proof system. The corresponding complexity class is ZK (resp., SZK).

Note that, in the help model, the dealer is computable in polynomial time given only the instance, and not a witness (hence the notation $D(x)$ ).

It is simple to show (by having the verifier simulate the dealer's help) that $\mathrm{ZK}^{\mathrm{h}}$ is contained in IP, the class of promise problems with interactive proofs:

Lemma 22. $\mathrm{ZK}^{\mathrm{h}} \subseteq \mathrm{IP}$.

\subsection{Statistical Zero Knowledge}

In this section, we state a few characterizations of statistical zero knowledge which will be related to the ones we will later obtain for the computational case. We begin by noting that, in the statistical case, Ben-Or and Gutfreund [7] showed that zero knowledge in the help model is equivalent to zero knowledge:

Theorem $23([7]) . \mathrm{SZK}^{\mathrm{h}}=\mathrm{SZK}$.

The theorem above implies that all the characterizations of SZK will also hold for $\mathrm{SZK}^{\mathrm{h}}$. In particular, $\mathrm{SZK}^{\mathrm{h}}$ shares the complete problems for SZK that are due to [36]35]33]:

Theorem 24 ([36 35, 33]). The following problems are SZK-complete: 
1. Statistical Difference:

$$
\begin{aligned}
& \mathrm{SD}_{Y}=\{(X, Y): \Delta(X, Y) \leq 1 / 3\} \\
& \mathrm{SD}_{N}=\{(X, Y): \Delta(X, Y) \geq 2 / 3\}
\end{aligned}
$$

where $X$ and $Y$ are samplable distributions specified by circuits that sample from them.

2. Entropy Difference:

$$
\begin{aligned}
& \operatorname{ED}_{Y}=\{(X, Y): \mathrm{H}(X) \geq \mathrm{H}(Y)+1\} \\
& \operatorname{ED}_{N}=\{(X, Y): \mathrm{H}(Y) \geq \mathrm{H}(X)+1\}
\end{aligned}
$$

where $X$ and $Y$ are samplable distributions specified by circuits that sample from them.

3. Conditional Entropy Approximation:

$$
\begin{aligned}
& \mathrm{CEA}_{Y}=\{(X, Y, r): H(X \mid Y) \geq r\} \\
& \mathrm{CEA}_{N}=\{(X, Y, r): H(X \mid Y) \leq r-1\}
\end{aligned}
$$

where $(X, Y)$ is a joint samplable distribution specified by circuits that use the same coin tosses.

Note that we can change the thresholds of $1 / 3$ and $2 / 3$ in SD to other thresholds $\alpha<\beta$. We denote the resulting problem $\mathrm{SD}^{\alpha, \beta}$. It is known that $\mathrm{SD}^{\alpha, \beta}$ is $\mathrm{SZK}$ complete for all constants $\alpha, \beta$ such that $0 \leq \alpha<\beta^{2} \leq 1$ [35].

\subsection{Computational Zero Knowledge}

In the case of ZK, no natural complete problems are known (unless we assume that one-way functions exist, in which case $\mathrm{ZK}=\mathrm{IP}=\mathrm{PSPACE}$ 417 18 38 39 20121]). However, characterizations that are analogous to the complete problems for SZK do exist in the form of the INDISTINGUISHABILITY CONdition and the Conditional Pseudoentropy Condition below. These conditions give 'if and only if' characterizations of ZK that provide essentially the same functionality that complete problems provide.

The first characterization is a natural computational analogue of STATISTICAL DIFFERENCE:

Definition 25. A promise problem $\Pi$ satisfies the INDISTINGUISHABILITY CONDITION if there is a polynomial-time computable function mapping strings $x$ to pairs of samplable distributions $(X, Y)$ such that:

- If $x \in \Pi_{Y}$, then $X$ and $Y$ are computationally indistinguishable.

- If $x \in \Pi_{N}$, then $\Delta(X, Y) \geq 2 / 3$.

Theorem 26 ([33]). $\Pi \in \mathrm{ZK}$ if and only if $\Pi \in \mathrm{IP}$ and $\Pi$ satisfies the INDISTINGUishaBiLity CONDITION.

The second characterization is based on the SZK-complete problem CEA: 
Definition 27. A promise problem $\Pi$ satisfies the Conditional PseudoenTROPY CONDITION if there is a polynomial-time computable function mapping strings $x$ to a samplable joint distribution $(X, Y)$ such that:

- If $x \in \Pi_{Y}$, then there exists a (not necessarily samplable) joint distribution $\left(X^{\prime}, Y^{\prime}\right)$ such that $\left(X^{\prime}, Y^{\prime}\right)$ is computationally indistinguishable from $(X, Y)$ and $H\left(X^{\prime} \mid Y^{\prime}\right) \geq r$.

- If $x \in \Pi_{N}$, then $H(X \mid Y) \leq r-1$.

Theorem 28 ([33]). $\Pi \in \mathrm{ZK}$ if and only if $\Pi \in \mathrm{IP}$ and $\Pi$ satisfies the ConDitional Pseudoentropy Condition.

Another characterization that we will use is the SZK/OWF Condition of [33]. The SZK/OWF CondiTion states that any problem in ZK can be decomposed into a part with an SZK proof and another part on which instance-dependent one-way functions can be constructed:

Definition 29 (SZK/OWF CONDITION [33). A promise problem $\Pi=$ $\left(\Pi_{Y}, \Pi_{N}\right)$ satisfies the $\mathrm{SZK} / \mathrm{OWF}$ Condition if there exists a set $I \subseteq \Pi_{Y}$ of YES such that:

1. The promise problem $\Pi^{\prime}=\left(\Pi_{Y} \backslash I, \Pi_{N}\right)$ is in $\mathrm{SZK}$.

2. There exists an instance-dependent one-way function on I (in the sense of Definition [6).

Theorem 30 ([33]). $\Pi \in \mathrm{ZK}$ if and only if $\Pi \in \mathrm{IP}$ and $\Pi$ satisfies the SZK/OWF CONDITION.

\section{Noninteractive Zero Knowledge}

\subsection{The Help Model}

In this section, we define the noninteractive analogue of zero-knowledge proofs in the help model.

Definition 31 (NIZK $^{\mathrm{h}}$, NISZK $^{\mathrm{h}}[7]$ ). $A$ noninteractive zero-knowledge proof system in the help model for a promise problem $\Pi$ is an interactive zeroknowledge proof in which there is only one message $\pi=P(x, \sigma)$ from prover to verifier.

If the real transcripts are statistically indistinguishable from simulated ones, we call it a noninteractive statistical zero knowledge proof system. NIZK ${ }^{\mathrm{h}}$ (resp., NISZK ${ }^{\mathrm{h}}$ ) is the class of promise problems possessing noninteractive zeroknowledge (resp., noninteractive statistical zero-knowledge) proof systems in the help model.

If the help string $\sigma$ is generated according to $D\left(1^{|x|}\right)$, we call the proof system $a$ noninteractive zero-knowledge proof system in the public parameter model. The corresponding complexity class is $\mathrm{NIZK}^{\mathrm{pub}}$ (resp., NISZK ${ }^{\mathrm{pub}}$ ). If the help string $\sigma$ is generated from the uniform distribution on $\{0,1\}^{|x|}$, we call the proof system an noninteractive zero-knowledge proof system in the common random string model. The corresponding complexity class is NIZK ${ }^{\mathrm{crs}}$ (resp., NISZK ${ }^{\mathrm{crs}}$ ). 
The main benefit of the public parameter model and the help model over the simpler CRS model is that they make it easier to construct NIZK proofs from simpler cryptographic primitives such as one-way functions $(7 \sqrt[23]{)})$, or, as we will show in this paper, from noninteractive, instance-dependent commitment schemes.

Like SZK, NISZK ${ }^{\mathrm{crs}}$ and NISZK ${ }^{\mathrm{h}}$ exhibit complete problems:

Theorem 32 ([25]). The promise problem Entropy Approximation, defined as:

$$
\begin{aligned}
& \operatorname{EA}_{Y}=\{(X, t): \mathrm{H}(X) \geq t+1\} \\
& \operatorname{EA}_{N}=\{(X, t): \mathrm{H}(Y) \leq t-1\}
\end{aligned}
$$

is complete for NISZK ${ }^{\mathrm{crs}}$, where $X$ is a samplable distribution specified by a circuit that samples from it. We use the notation $\mathrm{EA}^{t}$ to specify an instance of EA with parameter $t$.

Theorem $33([7])$. The promise problem Image Intersection Density, defined as:

$$
\begin{aligned}
& \operatorname{IID}_{Y}=\{(X, Y): \Delta(X, Y) \leq 1 / 3\} \\
& \operatorname{IID}_{N}=\{(X, Y): \operatorname{MutDisj}(X, Y) \geq 2 / 3\}
\end{aligned}
$$

is complete for $\mathrm{NISZK}^{\mathrm{h}}$, where $X$ and $Y$ are samplable distributions specified by circuits that sample from them.

We note that our definition of IID is slightly different than the one used by [7]. In our definition, we are working with mutual disjointness, since it is easy to transform disjoint distributions to mutually disjoint ones (Lemma 12). Additionally, due to a stronger Polarization Lemma that we will describe in a subsequent section, we use constant thresholds of $1 / 3$ and $2 / 3$ rather than functions tending to 0 and 1.

We also recall the complexity class AM, which is is the class of promise problems possessing constant-round interactive proofs, or equivalently, 2-round public-coin interactive proofs [14,15]. Analogous to Lemma 22, AM proves to be a natural upper bound for $\mathrm{NIZK}^{\mathrm{h}}$, since we can just have the verifier replace the dealer in creating the reference string. Also, a lower bound for $\mathrm{NIZK}^{\mathrm{h}}$ is NIZK $^{\mathrm{crs}}$, which is definitionally a more restricted version of the help model.

\section{Quantum Preliminaries and Definitions}

\subsection{The Quantum Formalism}

Let $\mathcal{H}$ denote a 2 -dimensional complex vector space, equipped with the standard inner product. We pick an orthonormal basis for this space, label the two basis 
vectors $|0\rangle$ and $|1\rangle$. A qubit is a unit length vector in this space, and so can be expressed as a linear combination of the basis states: $\alpha_{0}|0\rangle+\alpha_{1}|1\rangle$. Here $\alpha_{0}, \alpha_{1}$ are complex amplitudes, and $\left|\alpha_{0}\right|^{2}+\left|\alpha_{1}\right|^{2}=1$.

An $m$-qubit pure state is a unit vector in the $m$-fold tensor space $H \otimes \cdots \otimes H$. The $2^{m}$ basis states of this space are the $m$-fold tensor products of the states $|0\rangle$ and $|1\rangle$. For example, the basis states of a 2-qubit system are the four 4dimensional unit vectors $|0\rangle \otimes|0\rangle,|0\rangle \otimes|1\rangle,|1\rangle \otimes|0\rangle$, and $|1\rangle \otimes|1\rangle$. We abbreviate, e.g., $|1\rangle \otimes|0\rangle$ to $|0\rangle|1\rangle$, or $|1,0\rangle$, or $|10\rangle$, or even $|2\rangle$ (since 2 is 10 in binary). With these basis states, an $m$-qubit state $|\phi\rangle$ is a $2^{m}$-dimensional complex unit vector $|\phi\rangle=\sum_{i \in\{0,1\}^{m}} \alpha_{i}|i\rangle$. We use $\langle\phi|=| \phi\rangle^{*}$ to denote the conjugate transpose of the vector $|\phi\rangle$, and $\langle\phi \mid \psi\rangle=\langle\phi|\cdot| \psi\rangle$ for the inner product between states $|\phi\rangle$ and $|\psi\rangle$. These two states are orthogonal if $\langle\phi \mid \psi\rangle=0$. The norm of $|\phi\rangle$ is $\|\phi\|=\sqrt{\langle\phi \mid \phi\rangle}$.

A mixed state $\left\{p_{i},\left|\phi_{i}\right\rangle\right\}$ is a classical distribution over pure quantum states, where the system is in state $\left|\phi_{i}\right\rangle$ with probability $p_{i}$. We can represent a mixed quantum state by the density matrix which is defined as $\rho=\sum_{i} p_{i}\left|\phi_{i}\right\rangle\left\langle\phi_{i}\right|$. Note that $\rho$ is a positive semidefinite operator with trace (sum of diagonal entries) equal to 1 . The density matrix of a pure state $|\phi\rangle$ is $\rho=|\phi\rangle\langle\phi|$.

A quantum system is called bipartite if it consists of two subsystems. We can describe the state of each of these subsystems separately with the reduced density matrix. For example, if the joint quantum state of two subsystems $A, B$ has the form $|\phi\rangle=\sum_{i} \sqrt{p_{i}}|i\rangle_{A}\left|\phi_{i}\right\rangle_{B}$, then the state of the subsystem $B$, i.e., the subsystem which contains only the second part of $|\phi\rangle$ is described by the (reduced) density matrix $\sum_{i} p_{i}\left|\phi_{i}\right\rangle\left\langle\phi_{i}\right|$.

A quantum state evolves by a unitary operation or by a measurement. A unitary transformation $U$ is a linear mapping that preserves the complex $\ell_{2}$ norm. If we apply $U$ to a state $|\phi\rangle$, it evolves to $U|\phi\rangle$. A mixed state $\rho$ evolves to $U \rho U^{\dagger}$.

The most general measurement allowed by quantum mechanics is specified by a family of positive semidefinite operators $E_{i}=M_{i}^{*} M_{i}, 1 \leq i \leq k$, subject to the condition that $\sum_{i} E_{i}=I$. Given a density matrix $\rho$, the probability of observing the $i$ th outcome under this measurement is given by the trace $p_{i}=\operatorname{Tr}\left(E_{i} \rho\right)=$ $\operatorname{Tr}\left(M_{i} \rho M_{i}^{*}\right)$. These $p_{i}$ are nonnegative because $E_{i}$ and $\rho$ are positive semidefinite and they also sum to 1 . If the measurement yields outcome $i$, then the resulting mixed quantum state is $M_{i} \rho M_{i}^{*} / \operatorname{Tr}\left(M_{i} \rho M_{i}^{*}\right)$. In particular, if $\rho=|\phi\rangle\langle\phi|$, then $p_{i}=\left\langle\phi\left|E_{i}\right| \phi\right\rangle=\| M_{i}|\phi\rangle \|^{2}$, and the resulting state is $M_{i}|\phi\rangle / \| M_{i}|\phi\rangle \|$. A special case is where $k=2^{m}$ and $B=\left\{\left|\psi_{i}\right\rangle\right\}$ forms an orthonormal basis of the $m$-qubit space. 'Measuring in the $B$-basis' means that we apply the measurement given by $E_{i}=M_{i}=\left|\psi_{i}\right\rangle\left\langle\psi_{i}\right|$. Applying this to a pure state $|\phi\rangle$ gives resulting state $\left|\psi_{i}\right\rangle$ with probability $p_{i}=\left|\left\langle\phi \mid \psi_{i}\right\rangle\right|^{2}$.

The trace norm of a matrix $A$ is denoted by $\|A\|$ and is equal to the trace of $|A|$, where $|A|=\sqrt{A^{\dagger} A}$ is the positive square root of $A^{\dagger} A$. For two density matrices $\rho_{1}, \rho_{2}$ we define their trace distance as the trace norm of the matrix $\rho_{1}-\rho_{2}$, i.e., $\left\|\rho_{1}-\rho_{2}\right\|$.

The von Neumann Entropy of a mixed quantum state $\rho$ with eigenvalues $\lambda_{i}$ is defined as $S(\rho)=-\sum_{i} \lambda_{i} \log \lambda_{i}$. 


\subsection{Quantum Interactive and Noninteractive Statistical Zero-Knowledge}

Quantum statistical zero knowledge proofs are a special case of quantum interactive proofs. We can think of a quantum interactive protocol $\langle P, V\rangle(x)$ as a series of circuits $\left(V_{1}(x), P_{1}(x), \ldots, V_{k}(x), P_{k}(x)\right)$ on the space $\mathcal{V} \otimes \mathcal{M} \otimes \mathcal{P}$. $\mathcal{V}$ are the verifier's private qubits, $\mathcal{M}$ are the message qubits and $\mathcal{P}$ are the prover's private qubits. $V_{i}(x)$ (resp. $\left.P_{i}(x)\right)$ represents the $i^{t h}$ action of the verifier (resp. the prover) during the protocol and acts on $\mathcal{V} \otimes \mathcal{M}$ (resp. $\mathcal{M} \otimes \mathcal{P}) . \beta_{i}$ corresponds to the state that appears after the $i^{\text {th }}$ action of the protocol. We define completeness and soundness exactly the same way as in the case of classical protocols. We say that a protocol $\langle P, V\rangle$ solves $\Pi$ if it has completeness greater than $2 / 3$ and soundness less than $1 / 3$.

In the zero knowledge setting, we also want that the verifier learns nothing from the interaction other than the fact that $x \in \Pi_{Y}$ when it is the case. The way it is formalized is that for $x \in \Pi_{Y}$, the verifier can simulate his view of the protocol. We are interested only in honest verifier protocols where the verifier and the prover use unitary operations, since by Watrous 40. we know that honest verifier with unitary operations is equivalent to cheating verifier (that is allowed to use any permissible operation).

Let $\langle P, V\rangle$ a quantum protocol and $\beta_{j}$ defined as before. The verifier's view of the protocol is his private qubits and the message qubits, view $\langle P, V\rangle(j)=$ $\operatorname{Tr}_{\mathcal{P}}\left(\beta_{j}\right)$. We also want to separate the verifier's view based on whether the last action was made by the verifier or the prover. We note $\rho_{0}$ the input state, $\rho_{i}$ the verifier's view of the protocol after $P_{i}$ and $\xi_{i}$ the verifier's view of the protocol after $V_{i}$.

Definition 34. A quantum protocol $\langle P, V\rangle$ has the zero knowledge property for $\Pi$ if there exists a quantum polynomial-time simulator $\sigma$ and a negligible function $\mu$ such that for every input $x \in \Pi_{Y}$ and $\forall j\left\|\sigma_{j}(x)-\rho_{j}\right\| \leq \mu(|x|)$.

Note that for a state $\sigma$ such that $\left\|\sigma-\rho_{i}\right\| \leq \mu(|x|)$ it is easy to see that $\sigma^{\prime}=$ $V_{i+1} \sigma V_{i+1}^{\dagger}$ is close to $\xi_{i+1}=V_{i+1} \rho_{i} V_{i+1}^{\dagger}$ in this sense that $\left\|\sigma^{\prime}-\xi_{i+1}\right\| \leq \mu(|x|)$. Therefore, in the definition we just need to simulate the $\rho_{i}$ 's. Also note that the simulation in the quantum case is done round by round which seems to be a weaker definition than in the classical case. However, since the message qubits are reused in every round, the notion of a transcript can not be defined in the quantum case.

Definition 35. $\Pi \in Q S Z K$ iff there exists a quantum protocol $\langle P, V\rangle$ that solves $\Pi$ and that has the zero-knowledge property for $\Pi$.

In the setting of quantum noninteractive statistical zero knowledge, first defined by Kobayashi [13], the prover and verifier share a maximally entangled state $\sum_{i}|i\rangle_{P}|i\rangle_{V}$ created by a trusted third party: the dealer $D$. Then the prover sends a single quantum message to the verifier. We can assume that the message from the dealer to the verifier goes into his private space $\mathcal{V}$. Hence, after the prover's message, the verifier's view $\rho_{1}$ also contains the message from the dealer.

In this setting, we define the zero knowledge property as follows: 
Definition 36. A quantum noninteractive protocol $\langle D, P, V\rangle$ has the zero knowledge property for $\Pi$ if there exists a quantum polynomial-time simulator $\sigma$ and a negligible function $\mu$ such that for every input $x \in \Pi_{Y}\left\|\sigma(x)-\rho_{1}\right\| \leq \mu(|x|)$.

Definition 37. $\Pi \in$ QNISZK iff, when the prover and verifier share the maximally entangled state $\sum_{i}|i\rangle_{P}|i\rangle_{V}$ created by the dealer $D$, there exists a quantum noninteractive protocol $\langle D, P, V\rangle$ that solves $\Pi$ and that has the zero-knowledge property for $\Pi$.

\section{Statistical Zero Knowledge}

\subsection{The Polarization Lemma}

Zero knowledge protocols usually require from promise problems some parameters that are exponentially close to 0 or 1 . Polarizations are reductions from promise problems with weak parameters to promise problems that can be solved by the protocols. For example, there is a polarization for the promise problem $\mathrm{SD}$ that transforms $\mathrm{SD}^{a, b}$ with $a^{2}>b$ to $\mathrm{SD}^{1-2^{-k}, 2^{-k}}$ for any $k=\operatorname{poly}(n)$ [35].

The best polarization that was known for IID was that $\operatorname{IID}^{1 / n^{2}, 1-1 / n^{2}}$ reduces to IID $^{2^{-k}, 1-2^{-k}}$ and henceforth $\operatorname{IID}^{1 / n^{2}, 1-1 / n^{2}}$ is complete for NISZK ${ }^{\mathrm{h}} 7$. We will show here that $\operatorname{IID}^{a, b}$ is complete for $\operatorname{NISZK}^{\mathrm{h}}$ with $b>a$ (where $a$ and $b$ are constants).

Lemma 38 (Polarization Lemma [735]). There exists an algorithm that takes a pair of distributions $\left(X_{0}, X_{1}\right)$ and parameters $n \in \mathbb{N}, 0 \leq \alpha<\beta \leq 1$, and outputs a pair of distributions $\left(Y_{0}, Y_{1}\right)$ such that:

1. $\Delta\left(X_{0}, X_{1}\right) \leq \alpha \Rightarrow \Delta\left(X_{0}, X_{1}\right) \leq 2^{-n}$.

2. $\operatorname{MutDisj}\left(X_{0}, X_{1}\right) \geq \beta \Rightarrow \operatorname{MutDisj}\left(Y_{0}, Y_{1}\right) \geq 1-2^{-n}$.

The algorithm runs in time poly $\left(\left|\left(X_{0}, X_{1}\right)\right|, n, \exp \left(\frac{\alpha \log (1 / \beta)}{\beta-\alpha}\right)\right)$.

Proof. Let $\lambda=\min \{\beta / \alpha, 2\}>1$.

We first apply Lemma 15 with $k=\log _{\lambda} 2 n$, obtaining two distributions which are either statistically $\alpha^{k}$ close, or have $\beta^{k}$ mutual disjointness.

Then, we apply Lemma 13] with $m=\lambda^{k} /\left(2 \beta^{k}\right) \leq 1 /\left(2 \alpha^{k}\right)$. This gives two distributions with either statistical difference at most $m \alpha^{k} \leq 1 / 2$, or mutual disjointness of at most $1-\left(1-\beta^{k}\right)^{m} \geq 1-e^{-\beta^{k} m}=1-e^{-\beta^{k} \cdot \lambda^{k} /\left(2 \beta^{k}\right)}=$ $1-e^{-\lambda^{k} / 2}=1-e^{-n}$.

Finally, we apply again Lemma 15 with parameter $n$ to get either statistical difference at most $2^{-n}$, or mutual disjointness at most $\left(1-e^{-n}\right)^{n} \geq 1-n e^{-n} \geq$ $1-2^{-n}$, for sufficiently large $n$.

The running time of the algorithm is poly $\left(\left|\left(X_{0}, X_{1}\right)\right|, n, k\right)$, where $k=$ $O(\log n /(\lambda-1))=O(\alpha \log n /(\beta-\alpha))$ and $m \leq 1 / 2 \cdot(2 / \beta)^{k}=$ $\exp \left(O\left(\frac{\alpha \log n \log (2 / \beta)}{\beta-\alpha}\right)\right)$. This gives the claimed running time if either $n=O(1)$ 
or if $\beta-\alpha=\Omega(1)$. Thus we can obtain the lemma by applying the transformation in two steps, first with $n^{\prime}=2$ to polarize to thresholds $\alpha^{\prime}=1 / 4$ and $\beta^{\prime}=3 / 4$, and then once more with the desired value of $n$.

This can be compared to the original Polarization Lemma of [35], which refers to statistical difference in Item 2 (rather than mutual disjointness), but only achieves polarization from thresholds such that $0 \leq \alpha<\beta^{2} \leq 1$, and for which it is known that the gap between thresholds is inherent for a natural class of transformations 41].

\subsection{SZK and NISZK ${ }^{\mathrm{h}}$ are Equivalent}

We show in this section that help and interaction are equivalent in the statistical zero knowledge setting.

\section{Theorem 39. SZK $=$ NISZK $^{\mathrm{h}}$}

The inclusion NISZK ${ }^{\mathrm{h}} \subseteq$ SZK was proven by Ben-Or and Gutfreund 7 , since the NISZK ${ }^{\mathrm{h}}$-complete problem IMAGE InTERSECTION Density (IID) trivially reduces to Statistical Difference (SD), the SZK-complete problem. In what follows, we prove the opposite inclusion by reducing the SZK-complete problem Entropy Difference (ED) to IID. Ben-Or and Gutfreund claimed to have proven this reduction in [22] but due to a flaw they retracted it in [7]. Their reduction from ED to IID was in fact only a reduction to SD. Still, part of our proof is inspired by their method.

In order to prove that $\mathrm{SZK} \subseteq \mathrm{NISZK}^{\mathrm{h}}$, we follow 25] and reduce the SZKcomplete problem ED to several instances of ENTROPY APPROXIMATION and its complement (EA and $\overline{\mathrm{EA}}$ ) using the following fact:

Fact 40 ([25]) Let $X^{\prime}=X^{\otimes 3}$ and $Y^{\prime}=Y^{\otimes 3}$. Let $n$ the output size of $X^{\prime}$ and $Y^{\prime}$. It holds that:

$$
\begin{aligned}
& (X, Y) \in \mathrm{ED}_{Y} \Leftrightarrow \forall t \in\{1, \ldots, n\}\left[\left(\left(X^{\prime}, t\right) \in \mathrm{EA}_{Y}\right) \vee\left(\left(Y^{\prime}, t\right) \in \overline{\mathrm{EA}}_{Y}\right)\right] \\
& (X, Y) \in \mathrm{ED}_{N} \Leftrightarrow \exists t \in\{1, \ldots, n\}\left[\left(\left(X^{\prime}, t\right) \in \mathrm{EA}_{N}\right) \wedge\left(\left(Y^{\prime}, t\right) \in \overline{\mathrm{EA}}_{N}\right)\right]
\end{aligned}
$$

We know that $\mathrm{EA} \in \mathrm{NISZK}^{\mathrm{h}}$ ( since by definition $\mathrm{NISZK}^{\mathrm{crs}} \subseteq \mathrm{NISZK}^{\mathrm{h}}$ ), so it remains to show the following two things:

1. $\overline{\mathrm{EA}} \in \mathrm{NISZK}^{\mathrm{h}}$ : in order to this, we reduce $\overline{\mathrm{EA}}$ to IID, inspired by Ben-Or and Gutfreund's attempt 22] to reduce ED to IID. This reduction relies on ideas from [2728].

2. NISZK ${ }^{\mathrm{h}}$ has certain boolean closure properties: this will allow us to reduce ED to a single instance of IID. Since IID and SD are closely related, we use similar techniques to the ones used in 27,29.

Note that our proof's structure is similar to the approach suggested by Goldreich et al. 25. for showing that NISZK ${ }^{\mathrm{crs}}=\mathrm{SZK}$. They proved that if NISZK ${ }^{\mathrm{crs}}=$ co-NISZK ${ }^{\mathrm{crs}}$ then NISZK ${ }^{\mathrm{crs}}=$ SZK. We show here that co-NISZK $^{\mathrm{crs}} \subseteq$ NISZK $^{\mathrm{h}}$, and using the closure properties, conclude that NISZK $^{\mathrm{h}}=\mathrm{SZK}$. 


\section{3 $\overline{\text { EA }}$ Belongs to NISZK ${ }^{\mathrm{h}}$}

In this section, we prove the following lemma:

Lemma 41. $\overline{\mathrm{EA}} \in \mathrm{NISZK}^{\mathrm{h}}$.

Proof. We will reduce $\overline{\mathrm{EA}}$ to IID, which is complete for NISZK ${ }^{\mathrm{h}}$.

Let $(X, t)$ an instance of $\overline{\mathrm{EA}}$. By artificially adding input gates or output gates to $X$, we can assume that $X$ has $m$ input and output gates. Let $k$ a large constant that will be specified later on and $X^{\prime}=X^{\otimes s}$ with $s=4 \mathrm{~km}^{2}$. Note that $X^{\prime}$ has $m^{\prime}=s \cdot m$ input and output gates and $\mathrm{H}\left(X^{\prime}\right)=s \cdot \mathrm{H}(X)$. We have:

\section{Fact 42}

1. $X^{\prime}$ is $\Delta$-flat with $\Delta=2 \sqrt{k} m^{2}$, where $s$ was chosen such that $s=2 \sqrt{k} \Delta$.

2. $\operatorname{Pr}\left[X^{\prime}\right.$ is $\sqrt{k} \Delta$-typical $] \geq 1-2^{-\Omega(k)}$.

Given $(X, t)$, we can create two distributions $Z$ as $Z^{\prime}$ as following

$Z$ : Choose $r \stackrel{R}{\leftarrow}\{0,1\}^{m^{\prime}}, x=X^{\prime}(r), h \stackrel{R}{\leftarrow} \mathcal{H}_{m^{\prime}+s t, m^{\prime}}, z \stackrel{R}{\leftarrow}\{0,1\}^{m^{\prime}}$. Return $(x, h, z)$.

$Z^{\prime}$ : Choose $r \stackrel{R}{\leftarrow}\{0,1\}^{m^{\prime}}, x=X^{\prime}(r), h \stackrel{R}{\leftarrow} \mathcal{H}_{m^{\prime}+s t, m^{\prime}}, u \stackrel{R}{\leftarrow}\{0,1\}^{s t}$. Return $(x,(h, h(r, u)))$.

Note that $Z^{\prime}$ is of the form $Z^{\prime}=\left(X^{\prime}, A\right)$. We write $A_{x}$ to denote the distribution of $A$ conditioned on $X^{\prime}=x$. Note that we can describe $A_{x}$ as follows :

$A_{x}$ : Choose $r \stackrel{R}{\leftarrow}\left(X^{\prime}\right)^{-1}(x), h \stackrel{R}{\leftarrow} \mathcal{H}_{m^{\prime}+s t, m^{\prime}}, u \stackrel{R}{\leftarrow}\{0,1\}^{s t}$ and return $(h, h(r, u))$.

Hence, we need to show that, when conditioning on $X^{\prime}=x$, we have either $\Delta\left(\mathcal{U}, A_{x}\right)$ small (on the YES instances) or $\operatorname{Disj}\left(\mathcal{U}, A_{x}\right)$ large (on the NO instances).

For $x \in \operatorname{Supp}\left(X^{\prime}\right)$, let $\operatorname{wt}(x)=\log \left|\left(X^{\prime}\right)^{-1}(x)\right|=m^{\prime}-\log \left(\frac{1}{\operatorname{Pr}\left[X^{\prime}=x\right.}\right)$. The number of different possible inputs $(r, u)$ that are hashed in $A_{x}$ is $2^{\mathrm{wt}(x)+s t}$. Using Fact 42, it is easy to see that, if $\mathrm{H}(X) \leq t-1$, then wt $(x)$ will be large with high probability, whereas, if $\mathrm{H}(X) \geq t+1$, then $\operatorname{wt}(x)$ will be small with high probability. We can now show the following two claims which will allow us to conclude the proof.

Claim. $(X, t) \in \overline{\mathrm{EA}}_{Y} \Rightarrow \Delta\left(Z, Z^{\prime}\right)=2^{-\Omega(k)}$.

Proof. For all $x \in \operatorname{Supp}\left(X^{\prime}\right)$ that are $\sqrt{k} \Delta$-typical, $\left|\log \left(\frac{1}{\operatorname{Pr}\left[X^{\prime}=x\right]}\right)-\mathrm{H}\left(X^{\prime}\right)\right| \leq$ $\sqrt{k} \Delta$. Hence,

$$
\operatorname{wt}(x) \geq m^{\prime}-s \cdot \mathrm{H}(X)-\sqrt{k} \Delta \geq m^{\prime}-s t+s-\sqrt{k} \Delta \geq m^{\prime}-s t+\sqrt{k} \Delta .
$$

Therefore, the number of inputs $(r, u)$ such that $X^{\prime}(r)=x$ and $u \in\{0,1\}^{s t}$ is greater than $2^{m^{\prime}+\sqrt{k} \Delta} \geq 2^{m^{\prime}+k}$. By the Leftover Hash Lemma (Lemma 20), $\Delta\left(\mathcal{U}, A_{x}\right)=2^{-\Omega(k)}$. By Fact 42 , the probability of a $\sqrt{k} \Delta$-typical $x$ is $1-2^{-\Omega(k)}$ and hence we can conclude that $\Delta\left(Z, Z^{\prime}\right)=2^{-\Omega(k)}$. 
Claim. $(X, t) \in \overline{\mathrm{EA}}_{N} \Rightarrow \operatorname{Disj}\left(Z, Z^{\prime}\right)=1-2^{-\Omega(k)}$.

Proof. For all $x \in \operatorname{Supp}\left(X^{\prime}\right)$ that are $\sqrt{k} \Delta$-typical, we have:

$$
\mathrm{wt}(x) \leq m^{\prime}-s \cdot \mathrm{H}(X)+\sqrt{k} \Delta \leq m^{\prime}-s t-s+\sqrt{k} \Delta \leq m^{\prime}-s t-\sqrt{k} \Delta .
$$

Therefore, the number of inputs $(r, u)$ such that $X^{\prime}(r)=x$ and $u \in\{0,1\}^{s t}$ is smaller than $2^{m^{\prime}-\sqrt{k} \Delta} \leq 2^{m^{\prime}-k}$. Since we hash at most $2^{m^{\prime}-k}$ values into $\{0,1\}^{m^{\prime}}$, we get only a $2^{-k}$ fraction of the total support and hence $\operatorname{Disj}\left(\mathcal{U}, A_{x}\right)=$ $1-2^{-\Omega(k)}$. By Fact 42 , the probability of a $\sqrt{k} \Delta$-typical $x$ is $1-2^{-\Omega(k)}$ and hence we can conclude that $\operatorname{Disj}\left(Z, Z^{\prime}\right)=1-2^{-\Omega(k)}$.

By taking $k$ a large enough constant, we can ensure that $(X, t) \in \overline{\mathrm{EA}}_{Y} \Rightarrow$ $\Delta\left(Z, Z^{\prime}\right) \leq 1 / 4$ and also $(X, t) \in \overline{\mathrm{EA}}_{N} \Rightarrow \operatorname{Disj}\left(Z, Z^{\prime}\right) \geq 3 / 4$.

The only thing that remains is to transform the disjointness in the NO instances to mutual disjointness. We first apply Lemma 12 to create distributions $(A, B)$ such that $\Delta(A, B) \leq 1 / 4$ or $\operatorname{Disj}(A, B) \geq 3 / 8$. Then, by the polarization Lemma shown in Subsection 6.1, we create distributions $\left(A^{\prime}, B^{\prime}\right)$ such that $(X, t) \in \overline{\mathrm{EA}}_{Y} \Rightarrow \Delta\left(A^{\prime}, B^{\prime}\right) \leq 1 / 3$ and $(X, t) \in \overline{\mathrm{EA}}_{N} \Rightarrow \operatorname{Disj}\left(A^{\prime}, B^{\prime}\right) \geq 2 / 3$.

In conclusion, we see that from $(X, t)$, we have created distributions $A^{\prime}, B^{\prime}$ in polynomial time such that :

$-(X, t) \in \overline{\mathrm{EA}}_{Y} \Rightarrow\left(A^{\prime}, B^{\prime}\right) \in \operatorname{IID}_{Y}$.

$-(X, t) \in \overline{\mathrm{EA}}_{N} \Rightarrow\left(A^{\prime}, B^{\prime}\right) \in \operatorname{IID}_{N}$.

Hence, $\overline{\mathrm{EA}}$ reduces to IID and from the completeness of IID for NISZK ${ }^{\mathrm{h}}$, we have $\overline{\mathrm{EA}} \in \mathrm{NISZK}^{\mathrm{h}}$.

\subsection{Closure Properties for NISZK ${ }^{\mathrm{h}}$}

We now prove some closure properties of NISZK $^{\mathrm{h}}$ that we will use to complete the proof of Theorem 39. Every promise problem $\Pi \in$ NISZK $^{\mathrm{h}}$ reduces to IID and hence, we just have to concentrate on this problem. Note that this problem is very similar to the SZK-complete promise problem SD and hence we use similar techniques to those developed in 2927 to show closure properties for SZK. In our case, we just need to show some limited closure properties that will be enough to prove that $\overline{\mathrm{ED}} \in \mathrm{NISZK}^{\mathrm{h}}$.

Definition 43. Let $\Pi$ some promise problem. We define $\operatorname{AND}(\Pi)$ to be the following promise problem:

$-\operatorname{AND}(\Pi)_{Y}=\left\{\left(x_{1}, \ldots, x_{k}\right): \forall i \in\{1, \ldots, k\} x^{i} \in \Pi_{Y}\right\}$.

$-\operatorname{AND}(\Pi)_{N}=\left\{\left(x_{1}, \ldots, x_{k}\right): \exists i \in\{1, \ldots, k\} x^{i} \in \Pi_{N}\right\}$.

Similarly, we define $\mathrm{OR}(\Pi)$ for a pair of instances of $\Pi$.

Definition 44. Let $\Pi$ a promise problem. We define $\mathrm{OR}(\Pi)$ to be the following promise problem: 
$-\operatorname{OR}(\Pi)_{Y}=\left\{\left(x_{1}, x_{2}\right): \exists i \in\{1,2\} x^{i} \in \Pi_{Y}\right\}$.

$-\mathrm{OR}(\Pi)_{N}=\left\{\left(x_{1}, x_{2}\right): \forall i \in\{1,2\} x^{i} \in \Pi_{N}\right\}$.

We show that NISZK ${ }^{\mathrm{h}}$ is closed under AND and OR.

Lemma 45. NISZK ${ }^{\mathrm{h}}$ is closed under AND.

Proof. Let $\Pi$ be in NISZK $^{\mathrm{h}}$ and $\left(x_{1}, \ldots, x_{k}\right)$ be an instance of $\operatorname{AND}(\Pi)$. We reduce $\Pi$ to the IID problem which means that we transform each $x_{i}$ into a pair of distributions $\left(X^{i}, Y^{i}\right)$ such that $x_{i} \in \Pi_{Y} \Rightarrow\left(X^{i}, Y^{i}\right) \in \operatorname{IID}_{Y}$ and $x_{i} \in \Pi_{N} \Rightarrow$ $\left(X^{i}, Y^{i}\right) \in \operatorname{IID}_{N}$. Let $X=X^{1} \otimes \cdots \otimes X^{k}$ and $Y=Y^{1} \otimes \cdots \otimes Y^{k}$. We first polarize each pair $\left(X^{i}, Y^{i}\right)$ to have statistical difference at most $1 / 3 k$ or mutual disjointness at least $2 / 3$. From Lemma 13. we can easily see that $\left(x_{1}, \ldots, x_{k}\right) \in$ $\operatorname{AND}(\Pi)_{Y} \Rightarrow(X, Y) \in \operatorname{IID}_{Y}$ and that $\left(x_{1}, \ldots, x_{k}\right) \in \operatorname{AND}(\Pi)_{N} \Rightarrow(X, Y) \in$ $\operatorname{IID}_{N}$, which concludes our proof.

Lemma 46. NISZK ${ }^{\mathrm{h}}$ is closed under OR.

Proof. Let $\Pi$ be in NISZK ${ }^{\mathrm{h}}$. Let $\left(x_{1}, x_{2}\right)$ be an instance of $\mathrm{OR}(\Pi)$. We reduce $\Pi$ to the IID problem which means that we transform each $x_{i}$ into a pair of distributions $\left(X^{i}, Y^{i}\right)$ such that $x_{i} \in \Pi_{Y} \Rightarrow\left(X^{i}, Y^{i}\right) \in \operatorname{IID}_{Y}$ and $x_{i} \in \Pi_{N} \Rightarrow$ $\left(X^{i}, Y^{i}\right) \in \operatorname{IID}_{N}$. We first polarize each pair $\left(X^{i}, Y^{i}\right)$ to have statistical difference at most $1 / 3$ or mutual disjointness at least $\sqrt{2 / 3}$. Now, consider the pair $(A, B)$ obtained by XORing $\left(X_{1}, Y_{1}\right)$ and $\left(X_{2}, Y_{2}\right)$ (in the sense of Lemma 14). Using this Lemma, we conclude that $\left(x_{1}, x_{2}\right) \in \mathrm{OR}(\Pi)_{Y} \Rightarrow(A, B) \in \operatorname{IID}_{Y}$ and that $\left(x_{1}, x_{2}\right) \in \mathrm{OR}(\Pi)_{N} \Rightarrow(A, B) \in \operatorname{IID}_{N}$.

\subsection{Putting It Together}

We can now prove that $\mathrm{SZK} \subseteq \mathrm{NISZK}^{\mathrm{h}}$ and hence conclude the proof of Theorem [39. In the language of the previous section, Fact 40 says that the SZK-complete problem $\mathrm{ED}$ reduces to $\mathrm{AND}(\mathrm{OR}(\overline{\mathrm{EA}}, \mathrm{EA}))$ via a standard Karp (i.e., manyone) reduction. Since EA and $\overline{\mathrm{EA}}$ are in NISZK $^{\mathrm{h}}$ (Lemma 41) and NISZK ${ }^{\mathrm{h}}$ is closed under AND and OR (Lemma 45] and 46), we conclude that ED $\in$ NISZK $^{\mathrm{h}}$ and that $\mathrm{SZK} \subseteq \mathrm{NISZK}^{\mathrm{h}}$.

An interesting corollary is the following new complete problem for SZK.

Corollary 47. IID is complete for SZK.

\section{Computational Zero Knowledge}

In this section, we extend the results presented in the previous section to computational zero knowledge. However, the techniques that we have used in the statistical case cannot be applied directly here, so we take a more indirect route to proving an equivalence for the computational case. We define the COMPUTATIONAL Image Intersection Density Condition (CIIDC), a natural computational analogue of IID in the style of the INDistinguishabiLity CONDITION and the 
Conditional Pseudoentropy Condition used in 33 (see Section 3.2), and prove that all problems in ZK satisfy the CIIDC, building on our proof that every problem in SZK reduces to IID. Next we want to show that every problem in AM satisfying the CIIDC is in NISZK ${ }^{\mathrm{h}}$. However, as the approach used in 7 to show IID is in NISZK ${ }^{\mathrm{h}}$ does not generalize to the computational case, following [33, we get around this difficulty by interpreting the COMPUTATIONAL ImaGe INTERSECTION Density Condition as a special type of commitment scheme that is sufficient for constructing NIZK ${ }^{\mathrm{h}}$ proofs. Hence, we show that any promise problem in $\mathrm{ZK} \cap \mathrm{AM}$ has a NIZK ${ }^{\mathrm{h}}$ proof. For the other direction, we prove that ZK equals $\mathrm{ZK}^{\mathrm{h}}$, a class which contains NIZK ${ }^{\mathrm{h}}$, concluding that $\mathrm{NIZK}^{\mathrm{h}}=\mathrm{ZK} \cap \mathrm{AM}$.

\subsection{The Computational Image Intersection Density Condition}

We define the Computational Image Intersection Density Condition, and show that any promise problem with a ZK proof satisfies this condition.

Definition 48 (Computational Image Intersection Density CondTION (CIIDC)). A promise problem $\Pi$ satisfies CIIDC if there is a polynomial time mapping from strings $x \in \Pi$ to two distributions $(X, Y)$ specified by circuits sampling from them such that

1. If $x \in \Pi_{Y}$, then $X$ and $Y$ are computationally indistinguishable.

2. If $x \in \Pi_{N}$, then $(X, Y)$ have mutual disjointness at least $1 / 3$.

Lemma 49. Every promise problem $\Pi \in \mathrm{ZK}$ satisfies CIIDC.

Proof. Since every problem $\Pi \in \mathrm{ZK}$ satisfies the SZK/OWF Condition, it follows that $\Pi$ can be decomposed into two promise problems, $\Gamma$ and $\Theta$, such that $\Pi=\Gamma \cup \Theta, \Gamma \in \mathrm{SZK}=$ NISZK $^{\mathrm{h}}$ and for $x \in \Theta$, instance-dependent one-way functions can be constructed.

On the instances $x$ in $\Gamma$, a reduction to IID gives a pair $\left(X_{0}, X_{1}\right)$ such that on $x \in \Gamma_{Y}, \Delta\left(X_{0}, Y_{0}\right)$ is close to 0 , and, on $x \in \Gamma_{N}, \operatorname{MutDisj}\left(X_{0}, X_{1}\right)$ is close to 1 . Informally, on the instances in $\Theta$, we apply 20] to the instance-dependent oneway function to obtain an instance-dependent pseudorandom generator $G_{x}(\cdot)$, and consider the pair $\left(Y_{0}, Y_{1}\right)$ obtained by comparing the output of $G_{x}(\cdot)$ to the uniform distribution. Note that on $x \in \Theta_{Y},\left(Y_{0}, Y_{1}\right)$ will be computationally indistinguishable, while on $x \in \Theta_{N}$, it will be disjoint (since $G_{x}(\cdot)$ has a small support), and hence mutually disjoint by Lemma 12.

Since it might not be possible to efficiently distinguish between instances in $\Gamma$ and those in $\Theta$, it is not sufficient to simply map $x$ to $\left(X_{0}, X_{1}\right)$ when $x \in \Gamma$, and to $\left(Y_{0}, Y_{1}\right)$ when $x \in \Theta$. Rather, we map $x$ to $(X, Y)=\operatorname{XOR}\left(\left(X_{0}, X_{1}\right),\left(Y_{0}, Y_{1}\right)\right)$, which satisfies the CIIDC (by a computational analogue of Lemma 14).

\subsection{Noninteractive, Instance-Dependent Commitments}

We begin by reviewing Ben-Or and Gutfreund's [7 proof that IID is in NISZK ${ }^{\mathrm{h}}$ and note that this proof cannot be replicated in the computational case to show 
that every $\Pi$ satisfying the CIIDC is in NISZK ${ }^{\mathrm{h}}$. Ben-Or and Gutfreund show that IID is in NISZK ${ }^{\mathrm{h}}$ by polarizing $\left(X_{0}, X_{1}\right) \in \operatorname{IID}$ to the distributions $\left(Y_{0}, Y_{1}\right)$, setting the help string to $\sigma=Y_{0}(r)$ and having $P$ prove to $V$ that $\sigma \in \operatorname{Supp}\left(Y_{1}\right)$ by sending a random preimage in $Y_{1}^{-1}(\sigma)$. However, this protocol may fail to even have completeness for promise problems satisfying CIIDC, since the images of $Y_{0}$ and $Y_{1}$ might even be disjoint, although they are computationally indistinguishable. Indeed, we do not expect to show that every problem satisfying CIIDC is in NIZK ${ }^{\mathrm{h}}$, since NIZK ${ }^{\mathrm{h}} \subseteq \mathrm{AM}$ but problems outside AM may satisfy CIIDC (indeed, if one-way functions exist, every promise problem satisfies the CIIDC). Thus, in showing an equivalence between interactive and noninteractive zero knowledge in the computational case, it is necessary to use a different approach. Following [33, we view IID/CIIDC as a kind of instance-dependent commitment scheme, and use it to implement the general construction of noninteractive zero-knowledge proofs for AM [19].

We show that promise problems that reduce to IID or that satisfy CIIDC have a natural form of noninteractive, instance-dependent commitment schemes. In particular, for a promise problem $\Pi$ which reduces to IID (resp., satisfies the CIIDC), the sender and the receiver can use the Polarization Lemma to obtain a pair of distributions $\left(Y_{0}, Y_{1}\right)$ that are statistically close on YES instances, and mutually disjoint on NO instances. To commit to a bit $b$, the sender draws $c$ from $Y_{b}$ and outputs $c$ as the commitment. To reveal $b$, the sender only needs to prove that $c$ is drawn from $Y_{b}$ by presenting to the receiver the randomness used in sampling from $Y_{b}$. Note that this binding property requires that the sender generates the commitments honestly. (Otherwise, it could always generate the commitment from the intersection of the supports, even if it negligibly small.) While assuming an honest sender is usually not suitable in applications of commitments, it turns out to be fine for constructing $\mathrm{NIZK}^{\mathrm{h}}$ proofs, because the dealer generates the commitments.

We note that this commitment-based approach can also be used as an alternate, more circuitous proof of NISZK $^{\mathrm{h}}=\mathrm{SZK}$, since our results regarding commitments apply to both IID and CIIDC. Hence, the definitions and theorems presented below will deal with both the statistical and computational variants.

We now give a formal definition of the noninteractive, instance-dependent commitment schemes we will be using:

Definition 50. A noninteractive, instance-dependent commitment scheme is a family $\left\{\operatorname{Com}_{x}\right\}_{x \in\{0,1\}^{*}}$ with the following properties:

1. The scheme $\mathrm{Com}_{x}$ proceeds in the stages: the commit stage and the reveal stage. In both stages, both the sender and the receiver share as common input the instance $x$. Hence we denote the sender and receiver as $S_{x}$ and, respectively, $R_{x}$, and we write $\operatorname{Com}_{x}=\left(S_{x}, R_{x}\right)$.

2. At the beginning of the commit stage, the sender $S_{x}$ receives as private input the bit $b \in\{0,1\}$ to commit to. The sender then sends a single message $c=S(x, b)$ to the receiver. 
3. In the reveal stage, $S_{x}$ sends a pair $(b, d)$, where $d$ is the decommitmentstring for bit $b$. Receiver $R_{x}$ either accepts or rejects based on inputs $x, b, d$ and $c$.

4. The sender $S_{x}$ and receiver $R_{x}$ algorithms are computable in time poly $(|x|)$, given the instance $x$.

5. For every $x \in\{0,1\}^{*}, R_{x}$ will always accept (with probability 1 ) if both $S_{x}$ and $R_{x}$ follow their prescribed strategy.

Security Properties. We now define the security properties of noninteractive, instance-dependent commitment schemes. These properties will be natural extensions of the hiding and binding requirements of standard commitments:

Definition 51. A noninteractive, instance-dependent commitment scheme $\operatorname{Com}_{x}=\left(S_{x}, R_{x}\right)$ is statistically (resp., computationally) hiding on $I \subseteq\{0,1\}^{*}$ if for every (resp., nonuniform PPT) $R^{*}$, the ensembles $\left.\left\{S_{x}(0)\right)\right\}_{x \in I}$ and $\left\{\left(S_{x}(1)\right\}_{x \in I}\right.$ are statistically (resp., computationally) indistinguishable.

For a promise problem $\Pi=\left(\Pi_{Y}, \Pi_{N}\right)$, a noninteractive, instance-dependent commitment scheme $\mathrm{Com}_{x}$ is statistically (resp., computationally) hiding on the YES instances if $\mathrm{Com}_{x}$ is statistically (resp., computationally) hiding on $\Pi_{Y}$.

Definition 52. A noninteractive instance-dependent commitment scheme $\operatorname{Com}_{x}=\left(S_{x}, R_{x}\right)$ is statistically (resp., computationally) binding for honest senders on $I \subseteq\{0,1\}^{*}$ if there exists a negligible function $\varepsilon$ such that for all $x \in I$, a computationally unbounded (resp., nonuniform PPT) algorithm $S^{*}$ succeeds in the following game with probability at most $\varepsilon(|x|)$ :

$S$ outputs a commitment $c$. Then, given the coin tosses of $S, S^{*}$ outputs pairs $\left(0, d_{0}\right)$ and $\left(1, d_{1}\right)$ and succeeds if in the reveal stage, $R_{x}\left(0, d_{0}, c\right)=$ $R_{x}\left(1, d_{1}, c\right)=$ ACCEPT.

For a promise problem $\Pi=\left(\Pi_{Y}, \Pi_{N}\right)$, a noninteractive, instance-dependent commitment scheme $\mathrm{Com}_{x}$ is statistically (resp., computationally) binding for honest senders on the YES instances if $\mathrm{Com}_{x}$ is statistically (resp., computationally) binding on $\Pi_{Y}$.

Having defined noninteractive, instance-dependent commitment schemes, we proceed to show that they are equivalent to IID (resp., CIIDC), and consequently, SZK (resp., ZK).

Lemma 53. A promise problem $\Pi$ has a noninteractive, instance-dependent commitment scheme that is statistically (resp., computationally) hiding on YES instances and statistically binding for honest senders on NO instances if and only if $\Pi$ reduces to IID (resp., if and only if $\Pi$ satisfies the CIIDC).

Proof. For the backwards direction, consider a problem $\Pi$ that reduces to IID (the computational case will be similar). We construct the following protocol: 


\section{Commitment protocol for $\Pi$ :}

\section{Preprocessing:}

First, reduce $x \in \Pi$ to an instance $\left(X_{0}, X_{1}\right)$ of IID. Use the Polarization Lemma on $\left(X_{0}, X_{1}\right)$ to obtain $\left(Y_{0}, Y_{1}\right)$ such that, if $x \in \Pi_{Y}, \Delta\left(Y_{0}, Y_{1}\right) \leq 2^{-n}$, and, if $x \in \Pi_{N},\left(Y_{0}, Y_{1}\right)$ have mutual disjointness $\left(1-2^{-n}\right)$, where $n=|x|$.

\section{Commit Stage:}

$S_{x}(x, b)$ : To commit to bit $b \in\{0,1\}$, choose $d \stackrel{R}{\leftarrow}\{0,1\}^{m}$, where $m$ is the input length of $Y_{b}$, set $c=Y_{b}(d)$ and output $(c, d)$.

\section{Reveal Stage:}

$R_{x}(x, c, b, d):$ Accept if and only if $Y_{b}(d)=c$.

On $x \in \Pi_{Y}$, we know that $Y_{0}$ and $Y_{1}$ have negligible statistical difference. Hence, a commitment to 1 is statistically indistinguishable from a commitment to 0 . Hence, the scheme is computationally hiding on YES instances (actually, the scheme is statistically hiding.)

When $x \in \Pi_{N}$, the pair $\left(Y_{0}, Y_{1}\right)$ has mutual disjointness $\left(1-2^{-n}\right)$. It directly follows that only a negligible fraction of commitments can be opened in two ways.

In the case that we are working with a problem which satisfies the CIIDC, we use the same scheme. However, instead of polarizing, we will simply take direct products to amplify the mutual disjointness on NO instances while preserving computational indistinguishability on YES instances (Lemma 13).

For the forward direction, let $\operatorname{Com}_{x}=\left(S_{x}, R_{x}\right)$ be a noninteractive, instancedependent commitment scheme that is statistically hiding on YES instances and statistically binding for honest senders on NO instances, and consider $X=S_{x}(0)$ and $Y=S_{x}(1)$ :

- If $x \in \Pi_{Y}$, we know that $\Delta\left(\operatorname{view}_{R}\left(S_{x}(0), R\right)\right.$, $\left.\operatorname{view}_{R}\left(S_{x}(1), R\right)\right) \leq \varepsilon(|x|)$, and hence, $\Delta\left(S_{x}(0), S_{x}(1)\right) \leq \varepsilon(|x|)$.

- If $x \in \Pi_{N}$, assume that there exists no negligible function $\mu(|x|)$ such that $\operatorname{MutDisj}\left(S_{x}(0), S_{x}(1)\right)=(1-\mu(|x|))$. Hence for all negligible functions $\mu(|x|)$ and $c \leftarrow S_{x}(b), \operatorname{Pr}\left[c \in S_{x}(\bar{b})\right]>\mu(|x|)$. But then, $S$ can always succeed with probability greater than $\mu(|x|)$ at the game described in Definition 52. So, for some negligible $\mu,\left(S_{x}(0), S_{x}(1)\right)$ have mutual disjointness $(1-\mu(|x|))$, and $\Pi$ reduces to IID.

The proof for the computational case is analogous.

By combining our previous results concerning IID and CIIDC with Lemma 53, we obtain the following theorem:

Theorem 54. If a promise problem $\Pi$ is in $S Z K$ (resp., ZK), then $\Pi$ also has a noninteractive instance-dependent commitment scheme that is statistically (resp., computationally) hiding on YES instances and statistically binding for honest senders on $\mathrm{NO}$ instances.

Proof. This follows from the fact that any $\Pi \in$ SZK (resp., ZK) reduces to IID (resp., satisfies CIIDC) (Lemma 49). By Lemma 53, $\Pi$ has a noninteractive, instance-dependent commitment scheme. 


\subsection{From Noninteractive, Instance-Dependent Commitments to NIZK $^{\mathrm{h}}$}

In section, we will show that noninteractive, instance-dependent commitment schemes are sufficient to obtain NIZK ${ }^{\mathrm{h}}$. We start from the hidden bits model, a fictitious construction that implements noninteractive zero knowledge unconditionally for all promise problems in AM. Then, we show how our commitments can be employed in conjunction with this model to construct NIZK ${ }^{\mathrm{h}}$ proofs.

The Hidden Bits Model. The hidden bits model is a model due to Feige, Lapidot and Shamir [19] that allows for an unconditional construction of NIZK. It assumes that both the prover $P$ and the verifier $V$ share a common reference string $\sigma$, which we will call the hidden random string (HRS). However, only the prover can see the HRS. We can imagine that the individual bits of $\sigma$ are locked in boxes, and only the prover has the keys to unlock them. The prover can selectively unlock boxes and reveal bits of the hidden random string. However, without the prover's help, the verifier has no information about any of the bits in the HRS.

Definition 55 (NIZK in the Hidden Bits Model [19]). A noninteractive zero knowledge proof system in the hidden-bits model for a promise problem $\Pi$ is a pair of probabilistic algorithms $(P, V)$ (where $P$ and $V$ polynomial-time bounded) and a polynomial $l(|x|)=|\sigma|$, satisfying the following conditions:

1. Completeness. For all $x \in \Pi_{Y}, \operatorname{Pr}\left[\exists(I, \pi)\right.$ s.t. $\left.V\left(x, \sigma_{I}, I, \pi\right)=1\right] \geq \frac{2}{3}$, where $(I, \pi)=P(x, \sigma), I$ is a set of indices in $\{0, \ldots, l(k)\}$, and $\sigma_{I}$ is the sequence of opened bits of $\sigma,\left(\sigma_{i}: i \in I\right)$, and where the probability is taken over $\sigma \stackrel{R}{\longleftarrow}\{0,1\}^{l(|x|)}$ and the coin tosses of $P$ and $V$.

2. Soundness. For all $x \in \Pi_{N}$ and all $P^{*}, \operatorname{Pr}\left[\exists(I, \pi)\right.$ s.t. $\left.V\left(x, \sigma_{I}, I, \pi\right)=1\right] \leq \frac{1}{3}$, where $(I, \pi)=P^{*}(x, \sigma)$, where the probability is taken over $\sigma \stackrel{R}{\leftarrow}\{0,1\}^{l(|x|)}$ and the coin tosses of $P^{*}$ and $V$.

3. Zero Knowledge. There exists a PPT $S$ such that the ensembles of transcripts $\{(x, \sigma, P(x, \sigma))\}_{x}$ and $\{S(x)\}_{x}$ are statistically indistinguishable on $\Pi_{Y}$, where $\sigma \stackrel{R}{\leftarrow}\{0,1\}^{l(|x|)}$.

Note that we have defined the zero-knowledge condition in this model to be statistical rather than computational. Indeed, the known construction of hidden bits NIZK proof systems is unconditional and yields statistically indistinguishable proof systems.

Theorem 56 ([19]). Every promise problem $\Pi \in \mathrm{NP}$ has a hidden bits zero knowledge proof system $(P, V)$.

As has been observed before (e.g. 23), this construction for NP automatically implies one for all of AM.

Corollary 57 ([19]). Every promise problem $\Pi \in$ AM has a hidden bits zero knowledge proof system $(P, V)$. 
Proof. Informally, this result can be obtained by transforming an AM proof into a statement that there exists some message from the prover that the verifier accepts. Since this statement is an NP statement, it can be proven in the hidden bits NIZK model.

The corollary above shows that there exists an unconditional construction of NIZK for all problems in AM. However, this construction holds only in the impractical hidden bits model. In proving our results, we show how to implement this construction in the help model by exploiting a novel connection to noninteractive, instance-dependent commitment schemes:

Theorem 58. If $\Pi \in \mathrm{AM}$ and $\Pi$ has a noninteractive, honest-sender, instancedependent commitment scheme that is statistically (resp., computationally) hiding on YES instances and statistically binding for honest senders on NO instances, then $\Pi \in \mathrm{NISZK}^{\mathrm{h}}$ (resp., $\Pi \in \mathrm{NIZK}^{\mathrm{h}}$ ).

Proof. Our general strategy will be to exploit the correspondence between the algorithms in our definition of an instance-dependent commitment scheme, and the three algorithms in a NIZK ${ }^{\mathrm{h}}$ proof system. More specifically, we will have the dealer $D$ use the sender algorithm to commit to a hidden bits string (this is why we can afford to assume the sender is honest). Since the prover $P$ is allowed to be unbounded, we will use it to exhaustively search for openings to $D$ 's commitments. Finally, the verifier $V$ will use the receiver algorithm to check $P$ 's openings.

Let $\left(P^{\mathrm{HB}}, V^{\mathrm{HB}}\right)$ be a hidden bits proof system for $\Pi$ and let (Sen, Rec) be the noninteractive, honest-sender bit commitment scheme for $\Pi$. Then, the following proof system $(D, P, V)$ is $\mathrm{NIZK}^{\mathrm{h}}$ :

1. $D\left(x, 1^{k}\right)$ : Select $\sigma^{D} \stackrel{R}{\leftarrow}\{0,1\}^{m}$, and $\operatorname{run} \operatorname{Sen}\left(x, \sigma_{i}^{D}\right)$ to generate a commitment $c_{i}$, for all $i$. Output $c=\left(c_{1}, \ldots, c_{m}\right)$ as the public help parameter.

2. $P(x, c)$ : Exhaustively find a random opening $o_{i}^{P}$ for each $c_{i}$ (and, implicitly, each $\left.\sigma_{i}^{D}\right)$. If one commitment $c_{i}$ can be opened as both 0 or $1, P$ outputs $o_{i}^{P}$ according to the distribution $\left.O\right|_{C=c_{i}}$, where $(O, C)$ is the output of $S$ on a random bit $b$. Let $\sigma^{P}$ be the secret string obtained by $P$ opening $D$ 's help string. $P$ runs $P^{\mathrm{HB}}\left(x, \sigma^{P}\right)$ to obtain $(I, \pi)$. Send $\left(I, \sigma_{I}^{P}, o_{I}^{P}, \pi\right)$ to $V$.

3. $V\left(x, I, o_{I}^{P}, \pi\right)$ : Compute $\sigma_{j}^{P}, \forall j \in I$. Use Rec to check that the commitments are consistent. Run $V^{\mathrm{HB}}\left(x, I, \sigma_{I}^{P}, \pi\right)$ and accept if and only if $V^{\mathrm{HB}}$ accepts.

In the full version of the paper, we show that the construction above satisfies the completeness, soundness and zero knowledge properties, concluding that $\Pi$ is in NIZK ${ }^{\mathrm{h}}$.

\subsection{From $\mathrm{ZK}^{\mathrm{h}}$ to $\mathrm{ZK}$}

In this section, we generalize the results of Ben-Or and Gutfreund [7 that $\mathrm{SZK}^{\mathrm{h}}=\mathrm{SZK}$ (Theorem 23) to show that adding help to ZK proofs does not confer any additional power: 
Theorem 59 (Theorem 3, restated). $\mathrm{ZK}^{\mathrm{h}}=\mathrm{ZK}$.

To prove Theorem 23. Ben-Or and Gutfreund employ the techniques of 244236, by considering the output of the simulator $S$ for a zero-knowledge proof for $\Pi$ as the moves of a virtual prover and a virtual verifier. The simulated transcripts are compared to the transcripts output by a cheating strategy for a real prover $P_{S}$ (called the simulation-based prover), which tries to imitate the behavior of the virtual prover. Intuitively, on YES instances, the output of the simulator should be statistically close to the output of the simulationbased prover interacting with the real verifier. On NO instances, however, if we modify the simulator to accept with high probability (we can easily modify it to do that), the difference between the two transcripts must be significant. 7] exploit this to show that any problem in $\mathrm{SZK}^{\mathrm{h}}$ can be reduced to the intersection of the SZK-complete problems Statistical Difference([35]) and Entropy DIFFERENCE( 36$)$. Since the other direction $\left(\mathrm{SZK} \subseteq \mathrm{SZK}^{\mathrm{h}}\right)$ follows from the definitions, the conclusion that $\mathrm{SZK}=\mathrm{SZK}{ }^{\mathrm{h}}$ follows immediately. We will use the same strategy with $\mathrm{ZK}^{\mathrm{h}}$, replacing statistical measures of closeness with computational ones. To do this, we replace the SZK-complete problems SD and ED with the Indistinguishability Condition and the Conditional PseudoenTROPY CONDITION, which characterize the class ZK, and show that for every $\Pi \in \mathrm{ZK}^{\mathrm{h}}, \Pi$ can be reduced to the intersection of a problem which satisfies INDistinguishability Condition and a problem which satisfies Conditional Pseudoentropy Condition, and is thus in ZK.

\subsection{Putting It Together}

We can now use the previous sections' results to prove our main theorems regarding computational zero knowledge:

\section{Theorem 60 (Theorem 1, restated). $\mathrm{ZK}^{\mathrm{h}} \cap \mathrm{AM}=\mathrm{ZK} \cap \mathrm{AM}=\mathrm{NIZK}^{\mathrm{h}}$.}

Proof. By definition, NIZK ${ }^{\mathrm{h}} \subseteq \mathrm{ZK}^{\mathrm{h}} \cap \mathrm{AM}$. For the other direction, we know any $\Pi \in \mathrm{ZK}$ has a noninteractive, instance-dependent commitment scheme (Theorem [54), so a NIZK ${ }^{\mathrm{h}}$ proof can built for $\Pi$ (Theorem 58). Hence, $\mathrm{ZK}^{\mathrm{h}} \cap \mathrm{AM} \subseteq$ $\mathrm{NIZK}^{\mathrm{h}}$, which completes the proof of our theorem.

Theorem 61. $\Pi \in \mathrm{ZK}=\mathrm{ZK}^{\mathrm{h}}$ if and only if $\Pi \in \mathrm{IP}$ and $\Pi$ satisfies the CIIDC.

Proof. Since a promise problem that satisfies the CIIDC also satisfies the INDistinguishabILITY CONDITION (this follows from the fact that of two distributions have disjointness $\alpha$, they must have statistical difference at least $\alpha$ ), the promise problem must have a ZK proof system by Theorem 26. Conversely, any problem in $\mathrm{ZK}^{\mathrm{h}}=\mathrm{ZK}$ satisfies CIIDC by Lemma 49, 


\section{Quantum Statistical Zero Knowledge}

In this section, we study different variants of help for quantum noninteractive statistical zero knowledge. We start by providing complete problems for the class QNISZK defined by Kobayashi [13] and proceed to define the following two types of help: pure quantum help and mixed quantum help.

\subsection{Complete Problems for QNISZK}

Kobayashi [13] gave a complete problem for the class of quantum noninteractive perfect zero-knowledge, but not for statistical zero-knowledge. We continue this line of work and give two complete problems for QNISZK, QUANTUM ENtropy Approximation (QEA) and Quantum Statistical Closeness to UNIFORM (QSCU).

Let $\rho$ be a quantum mixed state of $n$ qubits which can be created in time polynomial in $n$ by a quantum machine and $t$ a positive integer. Then,

$$
\begin{array}{rlrl}
\mathrm{QEA}_{Y} & =\{(\rho, t): S(\rho) \geq t+1\} & & \operatorname{QSCU}_{Y}=\{\rho:\|\rho-\mathcal{U}\| \leq 1 / n\} \\
\operatorname{QEA}_{N}=\{(\rho, t): S(\rho) \leq t-1\} & \operatorname{QSCU}_{N}=\{\rho:\|\rho-\mathcal{U}\| \geq 1-1 / n\}
\end{array}
$$

Note that these problems are the quantum equivalents of EA and SCU where the statistical difference is replaced by the trace distance and the Shannon entropy by the von Neumann entropy.

Theorem 62. QEA and QSCU are complete for QNISZK.

Proof Sketch: We start by showing that QEA belongs to QNISZK by using results of Ben-Aroya and Ta-Shma (43]) on quantum expanders. Then, similarly to the classical case we reduce QSCU to QEA and last by Kobayashi's results ([13) we know that QSCU is hard for QNISZK. This concludes the proof.

\subsection{Help in Quantum Noninteractive Zero-Knowledge}

In quantum noninteractive zero knowledge, the only model we defined so far is the model where the prover and the verifier share the maximally entangled state $\sum_{i}|i\rangle_{P}|i\rangle_{V}$ which can be created by a dealer with quantum polynomial power (13]). In the previous section, we provided two complete problems for this class. Here, we extend this definition to allow the dealer to create as help a quantum state that depends on the input.

We define two types of help and study the resulting classes:

- Pure Help: In the usual framework of quantum zero-knowledge protocols, the prover and the verifier use only unitaries. We define QNISZK $^{\mathrm{h}}$ as the class where the prover and the verifier share a pure state (i.e., the outcome of a unitary operation) created by the dealer in quantum polynomial time. This state can depend on the input. Note that since the maximally entangled state is a pure state QNISZK $\subseteq$ QNISZK ${ }^{\mathrm{h}}$. In fact, we show that QNISZK ${ }^{\mathrm{h}}=$ $\mathrm{QSZK}=\mathrm{QSZK}^{\mathrm{h}}$. 
- Mixed Help: The previous definition does not allow the dealer to have some private coins and hence does not fully correspond to NISZK ${ }^{\mathrm{h}}$. We suppose now that the prover and verifier share a mixed quantum state created by the dealer. As before, the dealer has quantum polynomial power and the state depends on the input. We call the resulting class QNISZK ${ }^{\mathrm{mh}}$ and show that this kind of help is most probably stronger than quantum interaction.

For these classes, the definition of the zero knowledge property remains the same as in the case of QNISZK (Section 51).

Pure Help. We suppose here that there is a trusted dealer with quantum polynomial power. On input $x$, he performs a unitary $D_{x}$ and creates a pure state $D_{x}(|0\rangle)=\left|h_{P V}\right\rangle$ in the space $\mathcal{P} \times \mathcal{V}$. The prover gets $h_{P}=\operatorname{Tr}_{\mathcal{V}}\left(h_{P V}\right)$ and the verifier gets $h_{V}=\operatorname{Tr}_{\mathcal{P}}\left(h_{P V}\right)$. Note that the state $h_{P V}$ is a pure state and depends on the input.

Definition 63. We say that $\Pi \in \mathrm{QSZK}^{\mathrm{h}}$ (resp. $\Pi \in \mathrm{QNISZK}^{\mathrm{h}}$ ) if there is an interactive (resp. noninteractive) protocol $\langle D, P, V\rangle$ that solves $\Pi$, has the zero knowledge property and where the verifier and the prover share a pure state $h_{P V}$ created by a dealer $D$ that has quantum polynomial power and access to the input. They also start with an arbitrary polynomial number of qubits initialized at $|0\rangle$.

Next, we prove a quantum analogue of Theorem 39, i.e., interactive and noninteractive zero knowledge are equivalent in the pure help model. We remark that the proof of this statement is much more straightforward than in the classical case.

Theorem 64. QNISZK $^{\mathrm{h}}=\mathrm{QSZK}=\mathrm{QSZK}^{\mathrm{h}}$

Proof. We start by showing that QSZK $^{\mathrm{h}} \subseteq$ QSZK (and hence by definition $\left.\mathrm{QNISZK}^{\mathrm{h}} \subseteq \mathrm{QSZK}\right)$. Let $\Pi \in \mathrm{QSZK}^{\mathrm{h}}$ and $\langle D, P, V\rangle$ denote the protocol. Since $h_{P V}$ is a pure state, we can create another protocol $\langle\widetilde{P}, \widetilde{V}\rangle$ where the verifier takes the place of the dealer. That is, $V$ generates for his first message the state $\left|h_{P V}\right\rangle$ and sends the $h_{P}$ part to the dealer while keeping the $h_{V}$ part for himself. At this point, note that the verifier and prover have exactly the same states then when the dealer generates the state $\left|h_{P V}\right\rangle$ and sends it to them.

The protocol is the same so soundness and completeness are preserved. The first message in $\langle\widetilde{P}, \widetilde{V}\rangle$ can be simulated because the circuit of the dealer is public and computable in quantum polynomial time. The remaining messages in $\langle\widetilde{P}, \widetilde{V}\rangle$ can be simulated because of the zero-knowledge property of the protocol $\langle D, P, V\rangle$.

The inclusion $\mathrm{QSZK} \subseteq \mathrm{QNISZK}^{\mathrm{h}}$ (and hence by definition $\mathrm{QSZK} \subseteq \mathrm{QSZK}^{\mathrm{h}}$ ) follows immediately from Watrous' two-message protocol for the QSZK-complete problem QSD [12. The first message of the verifier can be replaced by the dealer's help. 
Mixed help. In the most general case, the dealer can create as help a mixed quantum state, i.e., a state that can depend on some private coins or measurements as well as the input.

Definition 65. We say that $\Pi \in \mathrm{QNISZK}^{\mathrm{mh}}$ if there is a noninteractive protocol $\langle D, P, V\rangle$ that solves $\Pi$ with the zero-knowledge property, where the verifier and the prover share a mixed state $h_{P V}$ created by a dealer $D$ that has quantum polynomial power and access to the input. They also start with $|0\rangle$ qubits.

Note that the only difference between QNISZK $^{\mathrm{h}}$ and QNISZK $^{\mathrm{mh}}$ is that the verifier and the prover share a mixed state instead of a pure state; however, we show that this difference is significant. In the classical case, a model was studied where the dealer flips some coins $r$ and sends correlated messages $m_{P}(r)$ and $m_{V}(r)$ to the prover and the verifier. The resulting class was called NISZK ${ }^{\mathrm{sec}}$ and it was shown by Pass and shelat in 23 that NISZK $^{\mathrm{sec}}=$ AM. To create the secret correlated messages $m_{P}(r)$ and $m_{V}(r)$ in our quantum setting, we just have to create the following state : $|\phi\rangle=\sum_{r}|r\rangle\left|m_{P}(r)\right\rangle\left|m_{V}(r)\right\rangle$. This state can be created in polynomial time because $m_{P}(r)$ and $m_{V}(r)$ can be created with a classical circuit. The dealer keeps the $r$ part, sends the $m_{P}$ part to the prover and the $m_{V}$ part to the verifier. From this construction, we can easily see that $\mathrm{AM}=\mathrm{NISZK}^{\mathrm{sec}} \subseteq \mathrm{QNISZK}^{\mathrm{mh}}$. Note that it is not known that NP $\subseteq$ QSZK $=$ QNISZK $^{\mathrm{h}}$ so this may be interpreted as evidence that QNISZK $^{\mathrm{h}}$ is a strict subset of QNISZK ${ }^{\mathrm{mh}}$.

Last, when we also allow the verifier to use non-unitary operations (i.e., private coins and measurements), we don't know if help and interaction are equivalent. The case of quantum zero knowledge protocols with non-unitary players is indeed very interesting and we refer the reader to 44 for more results.

Acknowledgements. We thank the anonymous referees for their helpful comments.

\section{References}

1. Chailloux, A., Kerenidis, I.: The role of help in classical and quantum zero-knowledge. Cryptology ePrint Archive, Report 2007/421 (2007), http://eprint.iacr.org/

2. Ciocan, D.F., Vadhan, S.: Interactive and noninteractive zero knowledge coincide in the help model. Cryptology ePrint Archive, Report 2007/389 (2007), http://eprint.iacr.org/

3. Ciocan, D.: Constructions and characterizations of non-interactive zero-knowledge. Undergradute thesis, Harvard University (2007)

4. Goldwasser, S., Micali, S., Rackoff, C.: The knowledge complexity of interactive proof systems. SIAM Journal on Computing 18(1), 186-208 (1989)

5. Blum, M., Feldman, P., Micali, S.: Non-interactive zero-knowledge and its applications (extended abstract). In: STOC 1988: Proceedings of the twentieth annual ACM symposium on Theory of computing, pp. 103-112 (1988)

6. Blum, M., De Santis, A., Micali, S., Persiano, G.: Noninteractive zero-knowledge. SIAM Journal on Computing 20(6), 1084-1118 (1991) 
7. Ben-Or, M., Gutfreund, D.: Trading help for interaction in statistical zeroknowledge proofs. Journal of Cryptology 16(2) (2003) (Preliminary version appeared as $[22])$

8. Brassard, G., Chaum, D., Crépeau, C.: Minimum disclosure proofs of knowledge. Journal of Computer and System Sciences 37(2), 156-189 (1988)

9. Nguyen, M.-H., Vadhan, S.: Zero knowledge with efficient provers. In: STOC 2006: Proceedings of the thirty-eighth annual ACM symposium on Theory of computing, pp. 287-295. ACM Press, New York (2006)

10. Ong, S.J., Vadhan, S.: Zero knowledge and soundness are symmetric. In: Naor, M. (ed.) EUROCRYPT 2007. LNCS, vol. 4515, Springer, Heidelberg (2007)

11. Kitaev, A., Watrous, J.: Parallelization, amplification, and exponential time simulation of quantum interactive proof systems. In: Proceedings of the 32nd ACM Symposium on Theory of computing, pp. 608-617 (2000)

12. Watrous, J.: Limits on the power of quantum statistical zero-knowledge. In: FOCS 2002: Proceedings of the 43rd Symposium on Foundations of Computer Science, Washington, DC, USA, pp. 459-468. IEEE Computer Society Press, Los Alamitos (2002)

13. Kobayashi, H.: Non-interactive quantum perfect and statistical zero-knowledge. In: Ibaraki, T., Katoh, N., Ono, H. (eds.) ISAAC 2003. LNCS, vol. 2906, pp. 178-188. Springer, Heidelberg (2003)

14. Babai, L., Moran, S.: Arthur-Merlin games: A randomized proof system and a hierarchy of complexity classes. Journal of Computer and System Sciences 36, 254-276 (1988)

15. Goldwasser, S., Sipser, M.: Private coins versus public coins in interactive proof systems. In: Micali, S. (ed.) Advances in Computing Research, JAC Press, Inc., vol. 5, pp. 73-90 (1989)

16. Goldreich, O., Micali, S., Wigderson, A.: Proofs that yield nothing but their validity, or All languages in NP have zero-knowledge proof systems. Journal of the Association for Computing Machinery 38(3), 691-729 (1991)

17. Impagliazzo, R., Yung, M.: Direct Minimum Knowledge Computations. In: Pomerance, C. (ed.) CRYPTO 1987. LNCS, vol. 293, pp. 40-51. Springer, Heidelberg (1988)

18. Goldreich, O., Håstad, J., Goldwasser, S., Micali, S., Rogaway, P., Kilian, J., BenOr, M.: Everything Provable Is Provable in Zero-Knowledge. In: Goldwasser, S. (ed.) CRYPTO 1988. LNCS, vol. 403, pp. 37-56. Springer, Heidelberg (1990)

19. Feige, U., Lapidot, D., Shamir, A.: Multiple non-interactive zero knowledge proofs under general assumptions. SIAM Journal on Computing 29(1), 1-28 (1999)

20. Håstad, J., Impagliazzo, R., Levin, L.A., Luby, M.: A pseudorandom generator from any one-way function. SIAM Journal on Computing 28(4), 1364-1396 (1999)

21. Naor, M.: Bit commitment using pseudorandomness. Journal of Cryptology 4(2), 151-158 (1991)

22. Gutfreund, D., Ben-Or, M.: Increasing the power of the dealer in non-interactive zero-knowledge proof systems. In: Okamoto, T. (ed.) ASIACRYPT 2000. LNCS, vol. 1976, pp. 429-442. Springer, Heidelberg (2000), (Journal version appeared as $[7])$

23. Pass, R., Shelat, A.: Unconditional characterizations of non-interactive zeroknowledge. In: Shoup, V. (ed.) CRYPTO 2005. LNCS, vol. 3621, pp. 118-134. Springer, Heidelberg (2005)

24. Aiello, W., Håstad, J.: Statistical zero-knowledge languages can be recognized in two rounds. Journal of Computer and System Sciences 42(3), 327-345 (1991) 
25. Goldreich, O., Sahai, A., Vadhan, S.: Can statistical zero-knowledge be made noninteractive?, or On the relationship of SZK and NISZK. In: Wiener, M.J. (ed.) CRYPTO 1999. LNCS, vol. 1666, pp. 467-484. Springer, Heidelberg (1999)

26. Goldreich, O., Sahai, A., Vadhan, S.: Honest verifier statistical zero-knowledge equals general statistical zero-knowledge. In: Proceedings of the 30th Annual ACM Symposium on Theory of Computing, pp. 399-408 (1998)

27. Sahai, A., Vadhan, S.: Manipulating statistical difference. In: Pardalos, P., Rajasekaran, S., Rolim, J. (eds.) Randomization Methods in Algorithm Design (DIMACS Workshop, December 1997. DIMACS Series in Discrete Mathematics and Theoretical Computer Science, vol. 43, pp. 251-270. American Mathematical Society (1999)

28. Okamoto, T.: On relationships between statistical zero-knowledge proofs. Journal of Computer and System Sciences 60(1), 47-108 (2000)

29. De Santis, A., De Crescenzo, G., Persiano, G., Yung, M.: On monotone formula closure of SZK. In: Proc. 26th ACM Symp. on Theory of Computing, Montreal, Canada, pp. 454-465. ACM, New York (1994)

30. Bellare, M., Micali, S., Ostrovsky, R.: Perfect zero-knowledge in constant rounds. In: STOC 1990: Proceedings of the twenty-second annual ACM symposium on Theory of computing, pp. 482-493 (1990)

31. Itoh, T., Ohta, Y., Shizuya, H.: A language-dependent cryptographic primitive. Journal of Cryptology 10(1), 37-49 (1997)

32. Micciancio, D., Vadhan, S.: Statistical zero-knowledge proofs with efficient provers: Lattice problems and more. In: Boneh, D. (ed.) CRYPTO 2003. LNCS, vol. 2729, pp. 282-298. Springer, Heidelberg (2003)

33. Vadhan, S.: An unconditional study of computational zero knowledge. SIAM Journal on Computing 36(4), 1160-1214 (2006) (Special Issue on Randomness and Complexity)

34. Ong, S.J., Vadhan, S.: An equivalence between zero knowledge and commitments, These proceedings (2008)

35. Sahai, A., Vadhan, S.: A complete problem for statistical zero knowledge. Journal of the ACM 50(2), 196-249 (2003)

36. Goldreich, O., Vadhan, S.: Comparing entropies in statistical zero-knowledge with applications to the structure of SZK. In: Proceedings of the Fourteenth Annual IEEE Conference on Computational Complexity, Atlanta, GA, pp. 54-73 (1999)

37. Impagliazzo, R., Levin, L.A., Luby, M. (Pseudo-random generation from one-way functions (extended abstracts)) 12-24

38. Shamir, A.: IP = PSPACE. Journal of the ACM 39(4), 869-877 (1992)

39. Lund, C., Fortnow, L., Karloff, H., Nisan, N.: Algebraic methods for interactive proof systems. Journal of the ACM 39(4), 859-868 (1992)

40. Watrous, J.: Zero-knowledge against quantum attacks. In: STOC 2006: Proceedings of the thirty-eighth annual ACM Symposium on Theory of Computing, pp. 296305. ACM Press, New York (2006)

41. Holenstein, T., Renner, R.: One-way secret-key agreement and applications to circuit polarization and immunization of public-key encryption. In: CRYPTO 2005, pp. 478-493. ACM Press, New York (2005)

42. Petrank, E., Tardos, G.: On the knowledge complexity of NP. In: IEEE Symposium on Foundations of Computer Science, pp. 494-503 (1996)

43. Ben-Aroya, A., Ta-Shma, A.: Quantum expanders and the quantum entropy difference problem. ArXiv Quantum Physics e-prints, quant-ph/0702129 (2007)

44. Chailloux, A., Kerenidis, I.: Increasing the power of the verifier in quantum zero knowledge. Arxiv Quantum Physics e-prints, quant-ph/07114032 (2007) 